\title{
Order-parameter fluctuations (OPF) in spin glasses: Monte Carlo simulations and exact results for small sizes
}

\author{
Marco Picco \\ LPTHE \\ Université Pierre et Marie Curie, PARIS VI \\ Université Denis Diderot, PARIS VII \\ Boite 126, Tour 16, $1^{\text {er }}$ étage, 4 place Jussieu \\ F-75252 Paris CEDEX 05, FRANCE \\ E-Mail: picco@lpthe.jussieu.fr \\ Felix Ritort and Marta Sales \\ Departament de Física Fonamental, Facultat de Física, Universitat de Barcelona \\ Diagonal 647, 08028 Barcelona (Spain) \\ E-Mail: ritort@ffn.ub.es,msales@ffn.ub.es
}

(October 26, 2018)

\begin{abstract}
The use of parametersmeasuring order-parameter fluctuations (OPF) has been encouraged by the recent results reported in 3 which show that two of these parameters, $G$ and $G_{c}$, take universal values in the $\lim _{T \rightarrow 0}$. In this paper we present a detailed study of parameters measuring OPF for two meanfield models with and without time-reversal symmetry which exhibit different patterns of replica symmetry breaking below the transition: the Sherrington-Kirkpatrick model with and without a field and the Ising $\mathrm{p}$-spin glass $(\mathrm{p}=3)$. We give numerical results and analyze the consequences which replica equivalence imposes on these models in the infinite volume. We give evidence for the transition in each system and discuss the character of finite-size effects. Furthermore, a comparative study between this new family of parameters and the usual Binder cumulant analysis shows what kind of new information can be extracted from the finite $T$ behavior of these quantities. The two main outcomes of this work are: 1) Parameters measuring OPF give better estimates than the Binder cumulant for $T_{c}$ and even for very small systems they give evidence for the transition. 2) For systems with no time-reversal symmetry, parameters defined in terms of connected quantities are the proper ones to look at.
\end{abstract}

\section{INTRODUCTION}

One of the main features of the low-temperature phase of spin-glasses is the lack of self-averageness of the order parameter. Parameters measuring order-parameter fluctuations (OPF), $A, A_{c}, G$, and $G_{c}$, where introduced to locate phase transitions in systems which did not contain time-reversal symmetry (TRS) in the Hamiltonianl. Very recently the use of these parameters has been enhanced in 2 (henceforth referred to as RS) where it has been shown that $G$ and $G_{c}$ take, in the low-temperature limit, the universal values $1 / 3$ and $13 / 31$ respectively, provided the ground state is unique and that there is no gap at zero field. This situation is generically met in spin-glasses with no gap at zero coupling, thus bringing more interest to the applicability of these parameters in the study of phase transitions.

In ordered systems, a good parameter to locate phase transitions is the Binder cumulant which is the kurtosis of the order-parameter probability distribution. The uniqueness of the ground state in that case is enough to guarantee that the Binder cumulant takes the universal value 1 at zero temperature for any finite volume. On the contrary, in order to find universal values for $G_{c}$ and $G$, one has to add a condition, namely the absence of gap in the local field distribution.

Concerning disordered systems, it is well known that the use of the Binder cumulant may present serious problems, specially in models without TRS in their Hamiltonian. Then, the Binder cumulant may take negative volues showing no evidence for a crossing of the different curves for different sizes. Recently, Hukushima and Kawamural have shown that this is specially true in models with one-step replica symmetry breaking where the Binder cumulant may take a negative value just below $T_{c}$. This explains the non-positiveness of the Binder cumulant in Potts glasses and $p$-spin models. The same being also true for $p$ even when there is time-reversal symmetryt.

However, at finite $T$, parameters which measure OPF always remain finite and positive. In systems with nonvanishing $\mathrm{OPF}$, the behavior of these parameters in the thermodynamic limit can be inferred from the restrictions that the replica equivalence property imposes on the $P(q), q$ being the overlap between two different replicas of the 
same system. Even though the behavior of $G$ is trivial, the behavior of $G_{c}$ is not, and depends on which kind of replica symmetry breaking pattern (RSB) characterizes the frozen phase. This knowledge can help us to distinguish among different systems and can also provide us with useful information about the spin-glass (SG) phase.

One of the main outcomes of RS is that already very small sizes may yield valuable information about the infinite volume spin-glass transition. This is confirmed by previous numerical studies of Ising spin glasses and p-spin

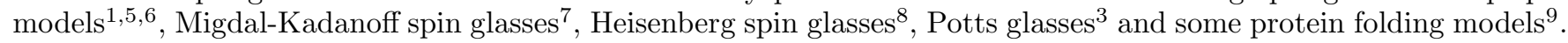
In view of the main results obtained in RS we will concentrate our attention on models where the new parameters $G$ and $G_{c}$, as well as $A$ and $A_{c}$ may yield valuable information not attainable using the Binder cumulant analysis. In particular, we are interested in models without time-reversal symmetry such as spin glasses in magnetic field and p-spin models.

Our purpose is to present a detailed numerical analysis of parameters which measure OPF by studying very small samples. Numerical calculations have been performed using exact computations of the partition function for small samples and Monte Carlo simulations. Monte Carlo calculations have been done in the spirit of the study on the spherical Sherrington-Kirkpatrick (SK) model shown in RS where very small sizes were simulated. One of the main outcomes of RS is that calculations of $G$ and $G_{c}$ at low-temperatures need a very large number of samples. This is due to the fact that at low temperatures only those samples having local fields much smaller than $T$ give a significant contribution to $G$ and $G_{c}$, so that the uncertainty in determining the value of $G$ and $G_{c}$ becomes very large. In other words, the low-temperature behavior of $G$ and $G_{c}$ provides direct information about the quality of the data. Having values for $G$ greater than $1 / 3$ shows either a lack of thermalization or poor sample statistics, a situation which the Binder cumulant does not reflect, since it is much less sensible to these fluctuations.

The paper is organized as follows. The next section presents the definitions used in this paper. Section III analyzes the consequences the replica equivalence imposes on the values of these parameters at finite $T$. Sections IV and V analyze two mean-field models exhibiting different kinds of RSB: the SK model with and without a magnetic field and the Ising $\mathrm{p}$-spin model with $\mathrm{p}=3$. Finally, in section VI, we present the conclusions. Two appendices are devoted to several technical points.

\section{A SHORT REMINDER}

Order-parameter fluctuations are an intrinsic characteristic of disordered systems. Although extensive quantities are self-averaging the order parameter may not. This is the typical situation found in systems where due to RSB there is a multiplicity of states and ergodicity is broken. Also in the case where replica-symmetry is not broken OPF are very sensitive to the spectrum of fluctuations around the stable solution. Spin glasses and disordered systems in general are characterized by the presence of large scale domain excitations without a typical length scale, and as a result correlations have a very slow decay, typically as a power law, and hence, with no characteristic length scale. The equilibrium phase is then marginally stable and OPF decay slower than $1 / V$ where $V$ is the volume. The possible scenarios concerning the behavior of OPF have been discussed in RS. Here we content ourselves with defining the parameters of interest in the analysis of this paper.

Four parameters which measure OPF were studied in RS: $G, G_{c}, A, A_{c}$, where the subindex $c$ stands for connected disorder quantities (to distinguish from disconnected quantities). $B$, the usual Binder cumulant and $B_{c}$, its connected counterpart were used in 10 to study the four dimensional spin glass with binary couplings. Calling $q=\frac{1}{V} \sum_{i} S_{i}^{a} S_{i}^{b}$ the spin overlap of two systems of spins $S_{i}^{a}$ and $S_{i}^{b}$, with $V$ the number of spins, we define these parameters as follows,

$$
\begin{aligned}
& B=\frac{1}{2}\left(3-\frac{\overline{\left\langle q^{4}\right\rangle}}{{\overline{\left\langle q^{2}\right\rangle}}^{2}}\right), \\
& A=\frac{\overline{\left\langle q^{2}\right\rangle^{2}}-{\overline{\left\langle q^{2}\right\rangle}}^{2}}{{\overline{\left\langle q^{2}\right\rangle}}^{2}}, \\
& G=\frac{\overline{\left\langle q^{2}\right\rangle^{2}}-{\overline{\left\langle q^{2}\right.}}^{2}}{\overline{\left\langle q^{4}\right\rangle}-{\overline{\left\langle q^{2}\right\rangle}}^{2}}=\frac{1}{2} \frac{A}{1-B}, \\
& B_{c}=\frac{1}{2}\left(3-\frac{\overline{\left\langle(q-\langle q\rangle)^{4}\right\rangle}}{\overline{\left\langle(q-\langle q\rangle)^{2}\right\rangle}}\right),
\end{aligned}
$$




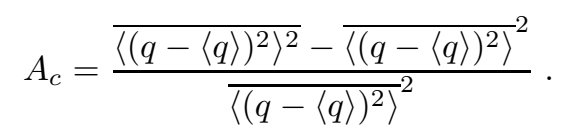

$$
\begin{aligned}
& G_{c}=\frac{\overline{\left\langle(q-\langle q\rangle)^{2}\right\rangle^{2}}-{\overline{\left\langle(q-\langle q\rangle)^{2}\right\rangle}}^{2}}{\overline{\left\langle(q-\langle q\rangle)^{4}\right\rangle}-{\overline{\left\langle(q-\langle q\rangle)^{2}\right\rangle}}^{2}}=\frac{1}{2} \frac{A_{c}}{1-B_{c}} .
\end{aligned}
$$

All of these parameters vanish at high temperature. It is well known that in the limit $T \rightarrow 0$ the Binder ratio takes the universal value 1, provided the ground state is unique. Int it was shown that under the hypothesis of a unique ground state and no gap in the local field distribution these parameters satisfy the following properties,

$$
\begin{aligned}
& \lim _{T \rightarrow 0} G(V, T)=\frac{1}{3} \\
& \lim _{T \rightarrow 0} G_{c}(V, T)=\frac{13}{31} \\
& \lim _{T \rightarrow 0} A(V, T)=0 .
\end{aligned}
$$

The last identity for $A$ follows trivially from (2) due to the uniqueness of the ground state. In Appendix A we make a brief summary of the main outcomes of section VI in RS to show how these universal values are obtained. The low-temperature results for $B_{c}$ and $A_{c}$ are also derived in that appendix. The results are:

$$
\lim _{T \rightarrow 0} A_{c}(V, T) \rightarrow \infty \quad, \quad \lim _{T \rightarrow 0} B_{c}(V, T) \rightarrow-\infty .
$$

These last two relations follow from the fact that even though numerator and denominator for both quantities vanish at zero temperature, the denominator vanishes as a higher power of $T$, and thus the ratio diverges.

We have to note that $A$ and $A_{c}$ have a non trivial behavior so they must be analyzed in detail in order not to extract wrong conclusions at low temperatures as shown by B. Drossel et al. in a very recent study of these parameters in the Migdal-Kadanoff approximation 11 In particular, one has to be careful when dealing with transitions at $T=0$, as the limits $T \rightarrow 0$ and $V \rightarrow \infty$ do not commute, so that results in (10) could not hold.

All these quantities are dimensionless, thus we expect that a finite-size scaling analysis can be performed 5 :

$$
X=f_{X}\left(L\left(T-T_{c}\right)^{1 / \nu_{X}}\right) \quad X=G, A, B .
$$

Quantities which can be obtained from derivatives of the free energy, i.e. thermodynamic quantities, are expected to have the same critical exponents, hence, for the Binder cumulant we have that $\nu_{B}=\nu$ (the usual correlation length exponent). However, we do not know a priori how to express $A, G$ in terms of thermodynamic quantities, so we cannot assure that scaling exponents for $G$ will be the same as the ones for $B$. The equivalent of (2) defined in terms of the magnetisation instead of $q$ has been extensively studied by numerical simulations for diluted model 12 and for the Ashkin-Teller random model13. In both cases the exponents associated with the parameters $A$ and $B$ ( $G$ is only function of $A, B$, see eq. (3)), within numerical precision, have been shown to be the same. Here we reach similar conclusions for the SK model.

Despite that critical behavior for $A, B, G$ is governed by the same exponents (i.e. by the critical behavior of the same correlation length) the parameters which measure OPF appear to be good indicators for a phase transition. In the spin-glass phase $G$ and $G_{c}$ attain a finite value and vanish in the paramagnetic phase. On the contrary, $A$ may vanish in both the spin-glass phase and paramagnetic phase but stays finite at $T_{c}$ (a result predicted for diluted systems 14 ). The interest of studying $A$ is that it does not vanish when OPF are finite, a situation characteristic of replica symmetry breaking transitions. Such information can not be deduced only from $G, G_{c}, B_{c}$ and $A_{c}$ which may be finite even when $\mathrm{OPF}$ vanish国.

Let us stress that only in cases where time-reversal symmetry is absent both class of parameters (connected or disconnected) can be studied. In case of models with time-reversal symmetry in the Hamiltonian only disconnected averages $(G, A, B)$ are feasible. A calculation of the disconnected parameters $G_{c}, A_{c}$ and $B_{c}$ would require to artificially break time-reversal symmetry which may impose additional difficulties to the simulation. This explains why in 3 only the parameters $G$ and $A$ were studied for the Ising spin glass chain and the spherical SK model. 


\section{REPLICA EQUIVALENCE}

Systems exhibiting RSB at the transition are well-known to have a non trivial $P(q)$ below the transition temperature. The information about this probability distribution is contaiped in the replica matrix $Q_{a b}$, where sub-indices stand for different replicas. The replica equivalence property (RE) 1517 states that every row/column of this matrix is a permutation of any other row. Thus, any quantity such as $\sum_{b} Q_{a b}$ does not depend on $a$ or, equivalently, the free energy must contain terms proportional to $n$ (which stands for the number of replicas) in order to ensure that there will be no divergences when performing the limit $n \rightarrow 01$, 19 .

This property imposes some constraints on the $P(q) \equiv \overline{P_{J}(q)}$. pne can derive general relations for the probability distributions of several overlaps and, as is pointed out in 15 , any probability function of $m$ overlaps $P\left(q_{12}, q_{34}, q_{56}, \ldots, q_{2(m-1) 2 m}\right)$ can be expressed in terms of the probability distribution of one overlap $P\left(q_{12}\right)$ plus the cyclic distributions, i.e. $P^{12,23,31}, P^{12,23,34,41}, . . P^{12,23,34, \ldots, m 1}$. For the case of two overlaps, fluctuations satisfy the following relation:

$$
P\left(q_{12}, q_{34}\right)=\frac{1}{3} P\left(q_{12}\right) \delta\left(q_{12}-q_{34}\right)+\frac{2}{3} P\left(q_{12}\right) P\left(q_{34}\right)
$$

which in the thermodynamic limit yields the well-known Guerra relations 20:

$$
\overline{\left\langle q_{12}^{2}\right\rangle^{2}}=\frac{1}{3} \overline{\left\langle q_{12}^{4}\right\rangle}+\frac{2}{3}{\overline{\left\langle q_{12}^{2}\right.}}^{2}
$$

which, as stressed in RS yield for systems with non-vanishing OPF (13) a trivial behavior of $G$, i.e.

$$
G=\frac{1}{3} \Theta\left(T-T_{c}\right)
$$

In RS it was pointed out that this result could hold even in spin-glass systems with a marginally stable replica symmetric phase. Notwithstanding, there is no such general trivial behavior for the other disconnected quantities, $A$ and $B$, neither for $G_{c}, A_{c}$ or $B_{c}$. To compute $G_{c}$ one has to deal with the probability of functions depending on three and four overlaps, so that in general one has a much more complicated object which depends on the following terms:

$$
\begin{aligned}
\frac{1}{n(n-1)} \sum_{a, b} Q_{a b}^{k} & =\int P(q) q^{k} d q \\
\frac{1}{n(n-1)(n-2)} \sum_{a, b, c} Q_{a b}^{k} Q_{b c} Q_{c a} & =\int P\left(q_{12}, q_{23}, q_{31}\right) q_{12}^{k} q_{23} q_{31} d q_{12} d q_{23} d q_{31} \\
\frac{1}{n(n-1)(n-2)(n-3)} \sum_{a, b, c, d} Q_{a b} Q_{b c} Q_{c d} Q_{d a} & =\int P\left(q_{12}, q_{23} q_{34}, q_{41}\right) q_{12}^{k} q_{23} q_{34} q_{41} d q_{12} d q_{23} d q_{34} d q_{41} \\
& \equiv \frac{\operatorname{Tr} Q^{4}}{n(n-1)(n-2)(n-3)} .
\end{aligned}
$$

Therefore, we expect that at finite $T$ the behavior of $G_{c}$ will depend on the kind of RSB that takes place at the transition, as it depends strongly on the structure of $Q_{a b}$. When computing the connected quantities, $A_{c}$ and $B_{c}$ one encounters the same situation so that we have to go to particular patterns of RSB in order to obtain a simpler expression for $G_{c}, A_{c}$ and $B_{c}$, as well as for $A$ and $B$.

In particular, for the case of one-step RSB, where $Q_{a b}$ can take two different values $q_{0}$ and $q_{1}$, and the $n$ replicas are divided in $m$ blocks, we obtain a very simple expression for all the connected quantities (calculations which lead to these results are shown in Appendix B):

$$
\begin{gathered}
G_{c}=\frac{39-113 m+98 m^{2}}{93-221 m+266 m^{2}}, \\
A_{c}=\frac{39-113 m+98 m^{2}}{140 m(1-m)}, \\
B_{c}=\frac{3\left(31-167 m+182 m^{2}\right)}{280 m(1-m)} .
\end{gathered}
$$


These results establish that connected quantities depend only on the RSB parameter $m$, which in general is a function of $T$. However, $A$ and $B$ depend also on the elements of the replica matrix, namely $q_{0}$ and $q_{1}$, as shown in the expressions (79) and (80) in Appendix B.

The simple functional dependence on $m$ of $A_{c}, G_{c}$ and $B_{c}$ lets us establish several universal features of these parameters. In first place, we note that below $T_{c}, G_{c}$ alfvays remains finite and positive and that at $T=0$, since $m=0$ in any system, it takes the universal value $13 / 31$. In the same way, at $m=1, G_{c}$ takes another universal value: $G_{c}=0.174$. Another interesting feature of $G_{c}$ is the existence of a local minimum at $m=0.650$ whose value $G_{c}=0.113$ is universal for all the systems exhibiting one-step RSB. As we will show in a forthcoming section, this is important from the point of view of numerical results for small sizes as the asymptotic position of this minimum can give us information about the behavior in the thermodynamic limit and thus about the dependence of $m$ on $T$.

In second place, we note that both $A_{c}$ and $B_{c}$ diverge at $m=0$, and therefore at zero temperature, the same being also true at $m=1$. The difference relies on the fact that while $A_{c}$ remains always positive and has a minimum at $m=0.56$ whose universal value is $A_{c}=0.187, B_{c}$ diverges to $-\infty$ and displays a maximum at a positive value $B_{c}=0.316(m=0.451)$. This is an interesting result, on the one hand because $G_{c}, A_{c}$ and $B_{c}$ provide us with valuable information about the characteristics of the SG phase, and on the other, because the exclusive dependence of the connected quantities on $m$ establishes a direct connection with models for structural glasses, where $m$, the RSB parameter, is well-known to control the violation of the fluctuation-dissipation theorem (FDT).

Unfortunately, for the two-step RSB scheme (and further RSB), we do not have such a simple expression and numerical computations for each system have to be performed. In the two following sections we analyze two meanfield models: the SK model in a field and the $p$-spin $(p=3)$ Ising model. Neither of the two models contains TRS in the Hamiltonian and exhibit RSB of different kinds: a full step and one-step RSB respectively. Therefore, these systems are characterized by having non-vanishing OPF and can provide a direct check of the results obtained in this section.

\section{THE SHERRINGTON-KIRKPATRICK MODEL WITH AND WITHOUT A MAGNETIC FIELD}

This section studies the Sherrington-Kirkpatrick model with and without a field. Preliminary results for the model in a field yere shown int. For the model without a field results have been recently presented by Hukushima and Kawamura 3. Here we present a more systematic and detailed study for small sizes of connected and disconnected quantities. The SK model in a field is defined by the disordered mean-field Hamiltonian,

$$
\mathcal{H}_{S K}=-\sum_{i<j} J_{i j} \sigma_{i} \sigma_{j}-h \sum_{i} \sigma_{i} .
$$

Before discussing the results in a field we discuss the case of zero field. An important differeme between the study of OPF parameters and Binder cumulants (for very precise recent SK simulation results see21.22) is that very highprecision statistics is needed to compute OPF parameters. Consider for instance $G$ and $G_{c}$. These are ratios of two quantities which may be very small if OPF vanish yielding a large error for $G$ and $G_{c}$. In models where OPF vanish simulating large sizes may then require a prohibitive computational effort. Not only long simulations are needed to thermalize the samples but high-precision statistics is needed to determine with reasonable precision the ratio of quantities which vanish in the infinite-volume limit. This second limitation is not present in models where OPF are finite such as the SK model at finite temperature.

\section{A. Zero-field simulations}

Results for $G$ and $A$ are shown in figures 1 and 2. In figure 1, we display results for rery small sizes $(N=4-11)$ obtained from exact computations of the partition function (such analysis was done in 23 for the SK model) averaging over 10000 samples of Gaussian quenched couplings with varianfe $1 / N$. In figure 2 we show results for larger sizes $(N=32,64,128,256,512)$ using the parallel tempering technique24 25 with binary couplings. The number of samples ranges from 1000 for the smallest size, $N=32$, to 250 for the biggest one, $N=512$. Figure 33 shows the Binder ratio $B$ for both groups of sizes. In figure 1 we show the results of the scaling analysis performed to the results for $B$ (a) and $G$ (b) for the larger sizes.

Monte Carlo simulations for larger sizes corroborate the results for $G$ and $A$ reported in 3 . It is important to note that, as this system contains TRS, the Binder cumulant for different sizes is expected to exhibit a crossing of the curves for different sizes as reported in 326 . However we must note that, while even for very small samples we do observe a crossing for $G$ and $A$ at $T$ close to $T_{c}=1$ (figure 1), for the same sizes, curves for $B$ do not display such 
a crossing (figure 3 (a)). In figure 3 we have plotted the Binder ratio for $N=32$ together with the smaller sizes to stress that we have to increase the size of the system (i.e. reach $N \approx 100$ ) to observe the crossing and not only a merging of all the curves in the low $T$ region. Results for larger systems display a crossing of the curves of all the parameters as expected. Our observations corroborate previous results which argued that finite-size corrections to $T_{c}$ for parameters measuring OPF were of opposite sign than for the Binder parameter. For $G$ and $A$ (figures 1 and 2) the crossing point at $T^{*}$ starts well above $T_{c}$ and approaches $T_{c}$ as we increase the size of the system. For the biggest sizes $T^{*}$ is very close to $T_{c}$. On the contrary, curves for $B$ cross at $T^{*}<T_{c}$, and therefore, since thermalization is more difficult at low $T$, the crossing is harder to observe. Moreover, the crossing for the biggest sizes takes place at a temperature slightly below $T_{c}$, thus making it evident that finite-size corrections to $T_{c}$ are much stronger in the Binder ratio than in $A$ and $G$.

The finite-size scaling analysis shown in figure 4 shows a remarkable result. In the particular case of the SK model, the argument of the scaling function (11) for the Binder ratio can be shown 27 to be $N\left(T-T_{c}\right)^{3}$. The collapse of the data, which are shown only for $T-T_{c}>1$, is very good, not only for $B$ but also for $G$ (the same being true for the parameter $A$, although not reported here). This suggests that exponents for $G$ are the same as for $B$, justifying a search for a general field-theoretical argument in the line of that proposed for diluted systems 14 .

As far as the details of the calculations are concerned, we have to point out that for small systems Gaussian couplings assure the uniqueness of the ground state, so that as reported in RS we obtain the universal values for $G$ and $G_{c}$. For binary couplings, however, this results should not hold for small systems, but as long as we increase the size of the system (i.e. we approach the thermodynamic limit) we should not expect a dependence on the bond distribution because in mean-field entropy vanishes at $T=0$ also in models with discrete couplings, and therefore, universal results should also hold. In order to be sure that results can be compared, we have checked that already for $N=33,32$ results for both coupling distributions nearly coincide.
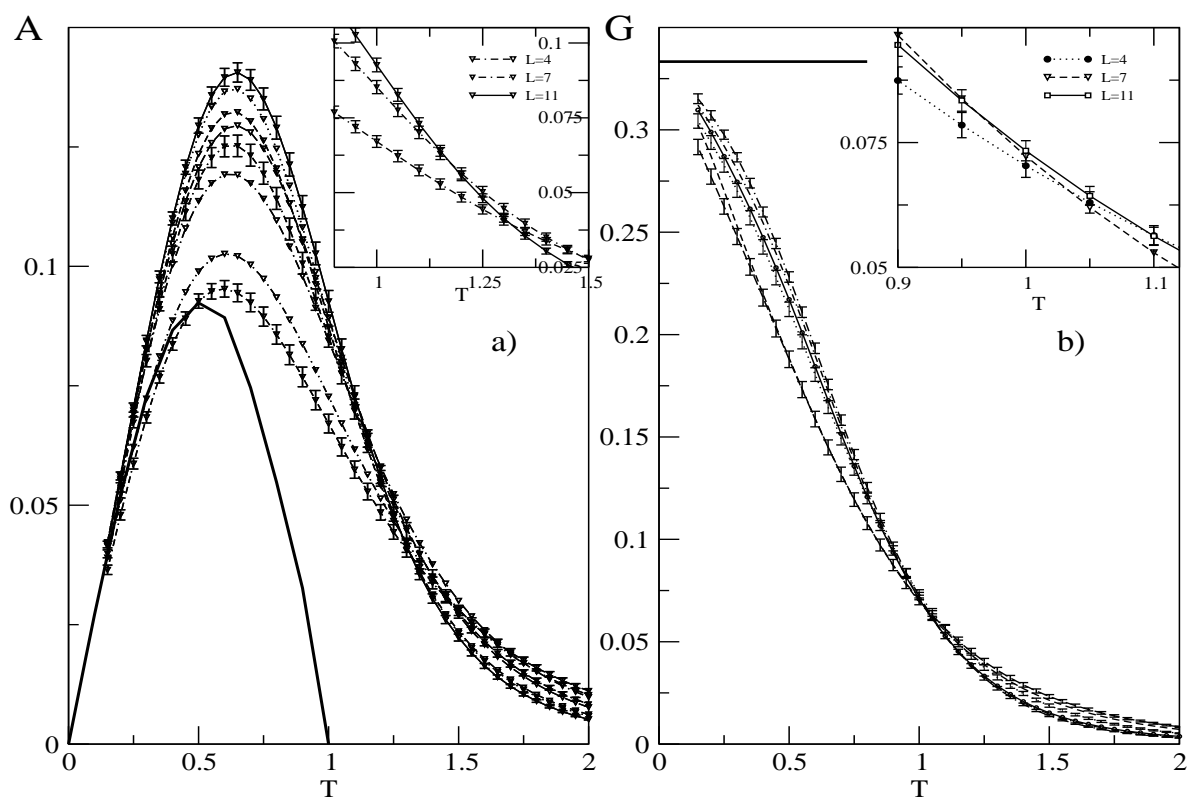

FIG. 1. Parameters $A(\mathrm{a})$ and $G$ (b) for the SK model with no field by exact computations of the partition function averaged over 10000 samples for $N=4,5,6,7,8,9,10,11$ from bottom to top at low $T$. Error bars are shown for sizes $N=4,7,11$. In both insets we show in detail the crossing region for sizes $N=4,7,11$. The solid line in (a) corresponds to numerical results of the one-step-approximation (79), obtained from solving numerically the saddle-point equations of the SK model for this particular case 28 . 

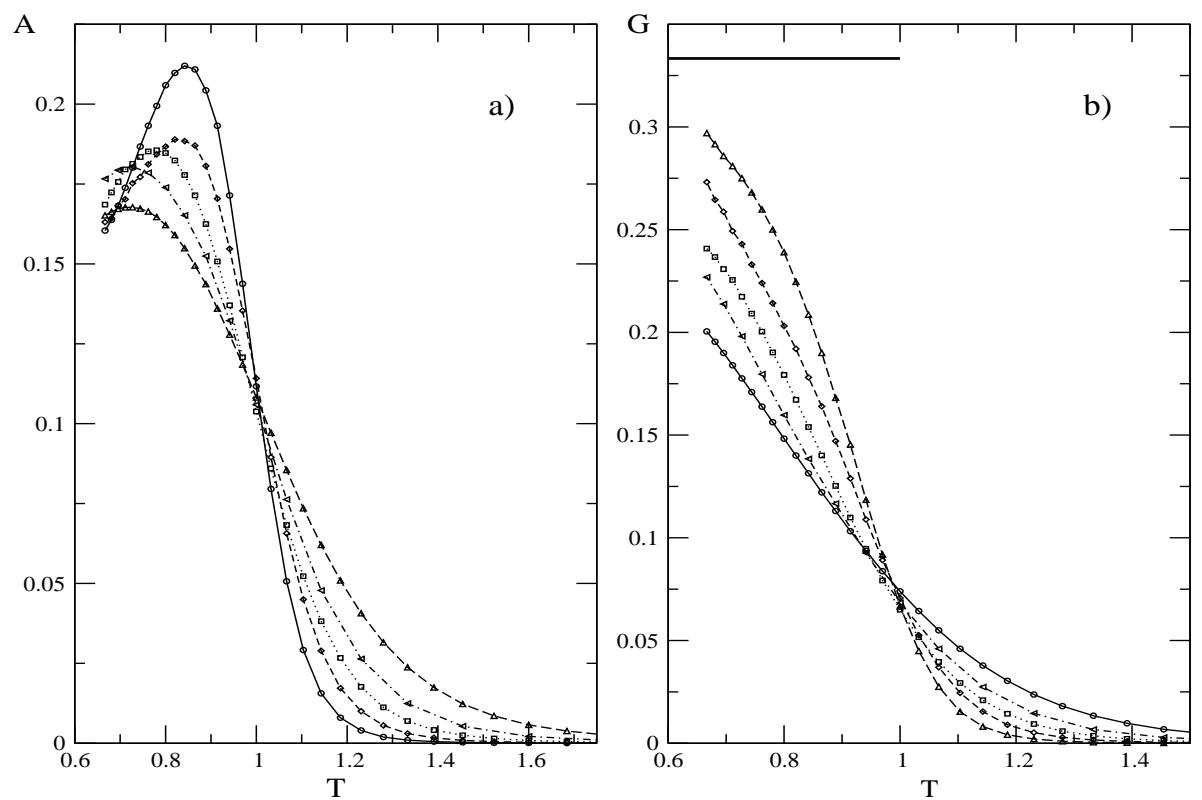

FIG. 2. Parameters $A$ (a) and $G$ (b) for the SK model with no field for the case of binary couplings for sizes $N=32,64,128,512$ from bottom to top in the high $T$ region.

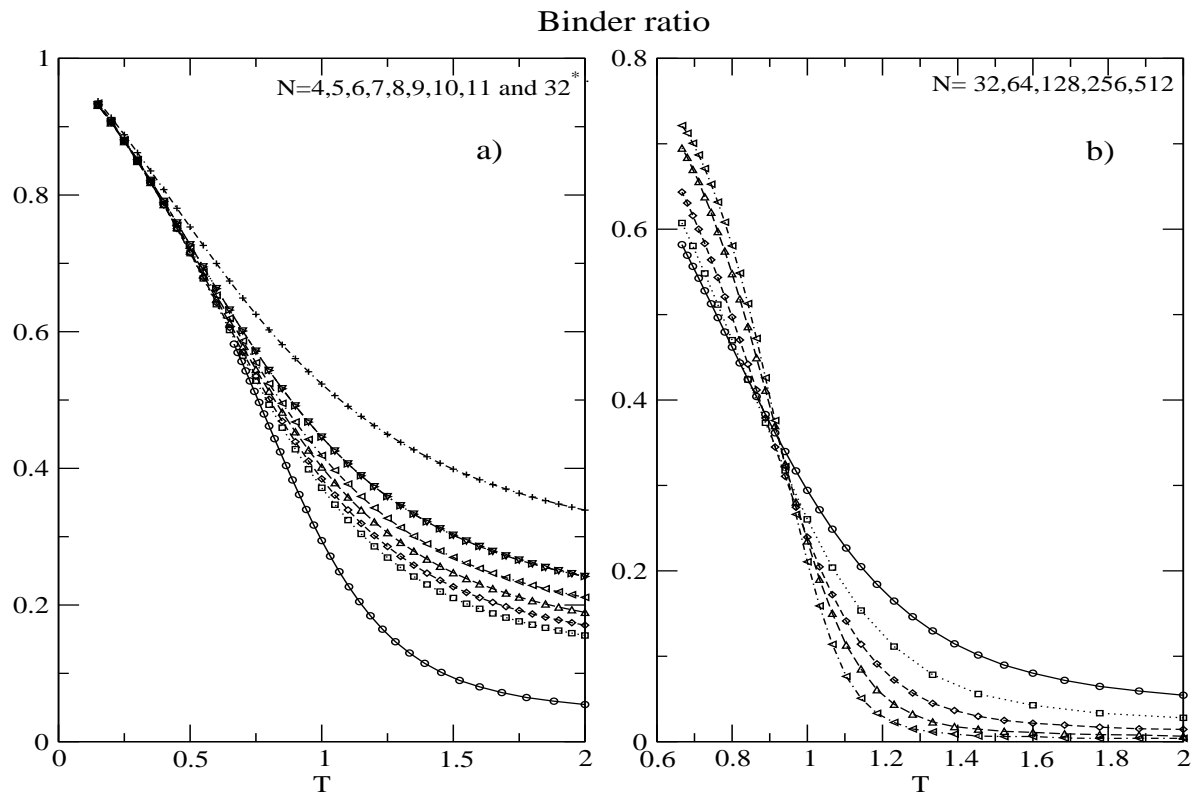

FIG. 3. Binder ratio, $B$, for the SK model with $h=0$ : (a) for small systems $(N=4,5,6,7,8,9,10,11$ from top to bottom) and (b) for $N=32,64,128,512$ from top to bottom at high $T$. 


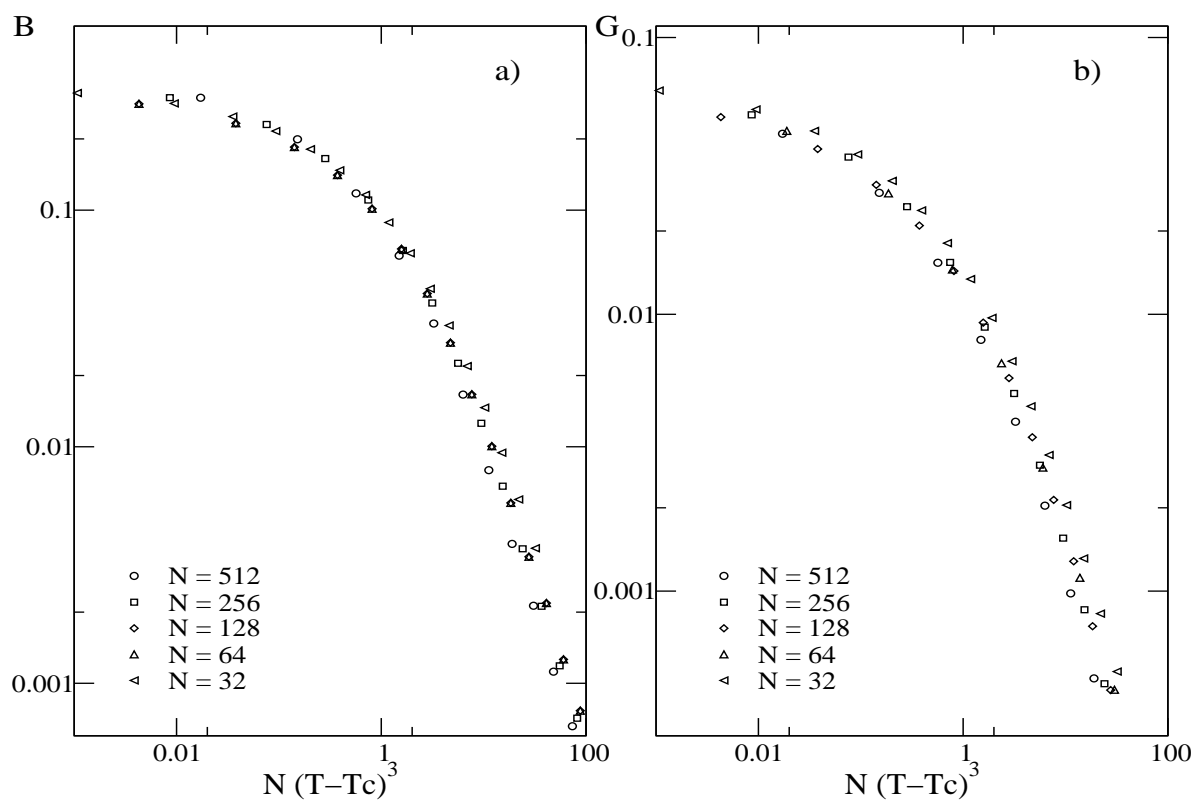

FIG. 4. Scaling functions of $B$ (a) and $G$ (b) for the SK model with $h=0$ for sizes $N=32,64,128,512$.

\section{B. Finite-field simulations}

In a field one must be a bit careful. According tol two conditions are necessary to ensure equations (7) and (8): uniqueness of the ground state and no gap in the local fields distribution. For the SK model with Gaussian couplings these conditions are immediately satisfied. But the first condition may be violated in the SK in a field. This happens in samples which have a ground state with zero magnetization. In this case the ground state is twofold degenerated because both the zero magnetization configuration and its fully reversed one have the same energy. In computing OPF for finite volume these rare samples have a finite probability violating the requirement of uniqueness of the ground state. Consequently eq. (8) for $G_{c}$ is not satisfied. Note that, on the contrary, eq. (7) is still satisfied because it is invariant against time-reversal symmetry. A zero magnetization ground state can only occur for samples with an even number of spins, so we expect strong differences for cases where $N$ is even or odd for small systems.

We have performed simulations with two applied fields $h=0.3$ and $h=0.6$. The transition into the frozen phase of the SK model in a field is determined by the de Almeida-Thouless line 28 which yields $T_{h=0.3}^{*}=0.65$ and $T_{h=0.6}^{*}=0.48$. In figure 5, we show results at field $h=0.3$ for an odd number of spins for sizes $N=3,5,7,9,11$ by exact calculation of the partition function and $N=13,17,23,33,64$ by Monte Carlo simulations. The largest size $(N=64)$ has been simulated with binary couplings and parallel tempering, while the smallest ones have been simulated by simple Metropolis algorithm with Gaussian distributed $J_{i j}$. As we have said at the beginning of this section, when systems are small and the ground state is not unique, eq. (8) is violated as can be observed in the inset of the plot for $G_{c}$ (figure (c)) where $G_{c}$ becomes greater than 13/31, and goes to 1 at $T=0$. This does not happen when systems are small and there is an odd number of spins. Results for $G_{c}$ and $G$ for the largest field, $h=0.6$ are shown in figure 6 for sizes $N=7,11,13,23,33$. Results for $A_{c}$, the Binder ratio and its corresponding connected quantity, $B_{c}$, are displayed in figures 7,8 and 9, for both applied fields, $h=0.3$ (a) and $h=0.6$ (b).

- Small fields: $h=0.3$.

First of all, let us focus our attention on the results for $h=0.3$. In figures f and 7 we observe that $G, G_{c}, A$ and $A_{c}$ display a clear crossing of the curves which is not seen neither for the Binder ratio where curves for all sizes seem to merge in the low temperature (figure 8 (a)), nor for the connected Binder cumulant (figure 9(a)). Despite, there is a clear difference between disconnected parameters $(G$ and $A)$ and connected ones $\left(G_{c}\right.$ and $\left.A_{c}\right)$. The crossing for disconnected quantities, $A$ and $G$, takes place at a temperature which is higher than 1 for small samples and slowly approaches 1 as we increase the size. On the contrary, results for $A_{c}$ and $G_{c}$, display a crossing, $T^{*}$, at a temperature which already for small samples is smaller than one, and that approaches the predicted $T_{c}=0.65$ as we increase the size of the system. Indeed, for the largest sizes $N=29,33,64$ curves cross around $T=0.65$ (figure 5 (c)). 

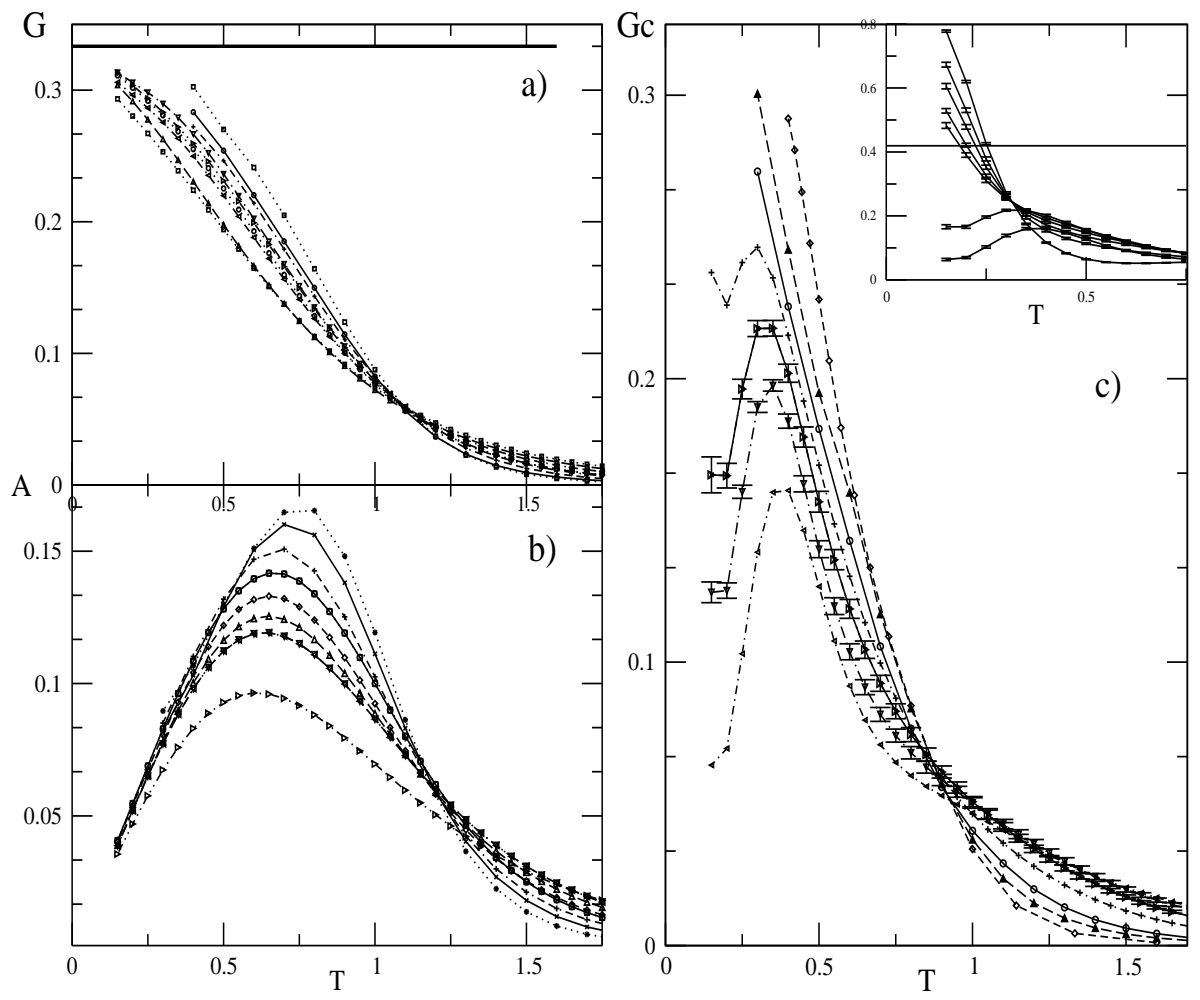

FIG. 5. Results for the $G, A$, and $G_{c}$ for the SK model in a field $h=0.3$. a) Curves for $G$ for $N=5,6,7,9,11-$ dashed lines -, 17, 23, 32, 64 from bottom to top at low $T$; the full line corresponds to the infinite-volume limit result $G=1 / 3$. b) Results for $A$, for sizes: $N=5,7,9,11,13,23,33$ (from bottom to top looking at the curves around $T \simeq 0.75$ ). c) Parameter $G_{c}$ for sizes: $N=3,5,7,9,11,23,32,64$. (Note that in c) there's no full line standing for the $T=0$ value of $G_{c}$ as it falls out of scale.) In the inset on the top right corner, we show the low $T$ behavior of $G_{c}$ for small samples with an even number of spins $N=2,4,6,8,10$ from top to bottom, the full line corresponds to the $T=0$ value $G_{c}=13 / 31$. Curves below the line correspond to samples with odd number of spins $N=3,5$.

- Large fields: $h=0.6$.

Results in a larger field, $h=0.6$, for $G, G_{c}$ and $A_{c}$ displayed in figures 6 and 7 (b) show a greater difference between $G$ and $G_{c}$. While the crossing for $G$ takes place at very high temperatures (close to 2), curves for $G_{c}$ cross at a temperature which even for small samples is close to $T_{c}=0.48$ and gets closer to it as we increase the size. From the Binder cumulant (figure 8 (b)) one gets no clear information. Numerical results display a crossing at high temperature as curves for $G$ do, but the crossing point seems to be moving towards higher temperatures, which suggests that this crossing has no real connection with the existence of a transition. Still, curves for $B_{c}$ (figure 9 (b)) show an interesting result, since we observe a crossing around $T^{*}=0.5$. Unfortunately, results are not very clean because the transition takes place at a very low temperature and thermalization is not easy. For this reason the crossing of curves for $A_{c}$ in figure 7 (b) is blurred by the existence of the divergence at $T=0$. Notwithstanding this, our results, together with the results in 30 for the $P(q)$, which for very large sizes presented two peaks in the positions predicted by the RSB solution, are the most clear evidence for a transition in such a high field $(h=0.6)$ which has been reported up to now. 

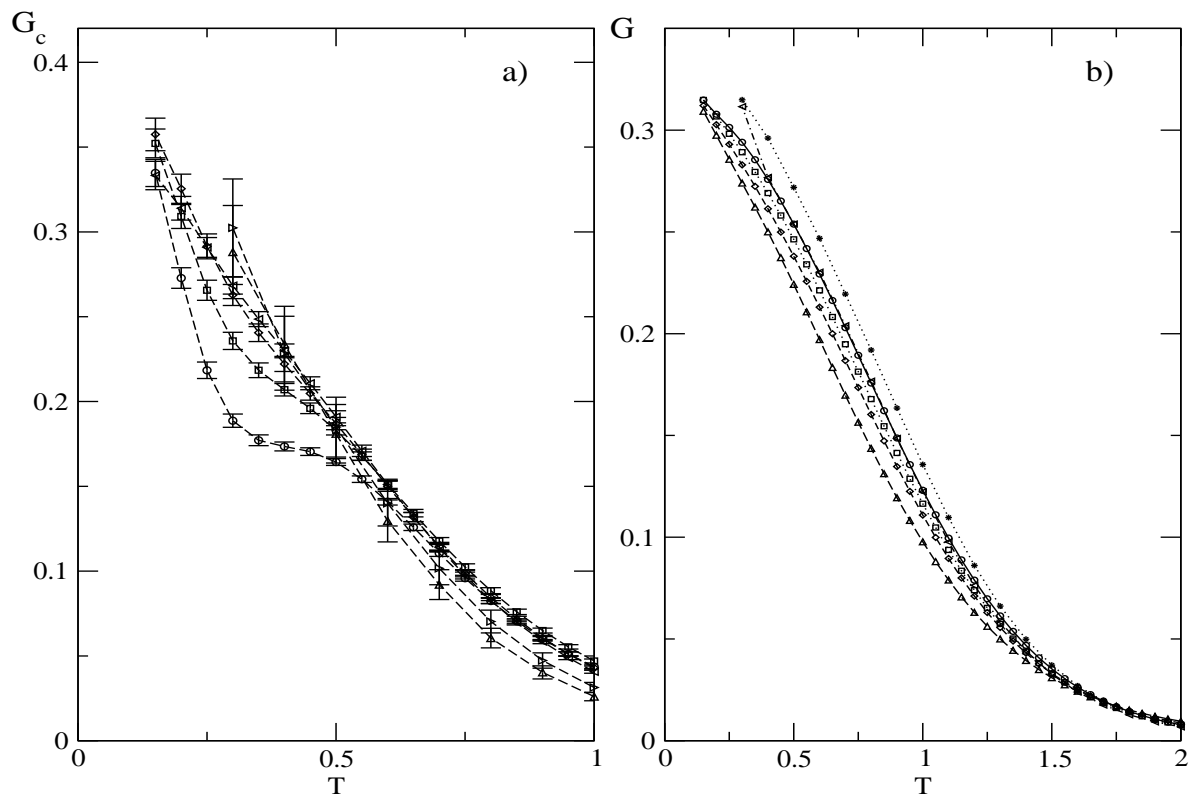

FIG. 6. $G_{c}$ (a) and $G$ (b) for the SK model in a field $h=0.6$ for sizes $N=5,7,9,11,17,29$ from bottom to top in the low $T$ region.

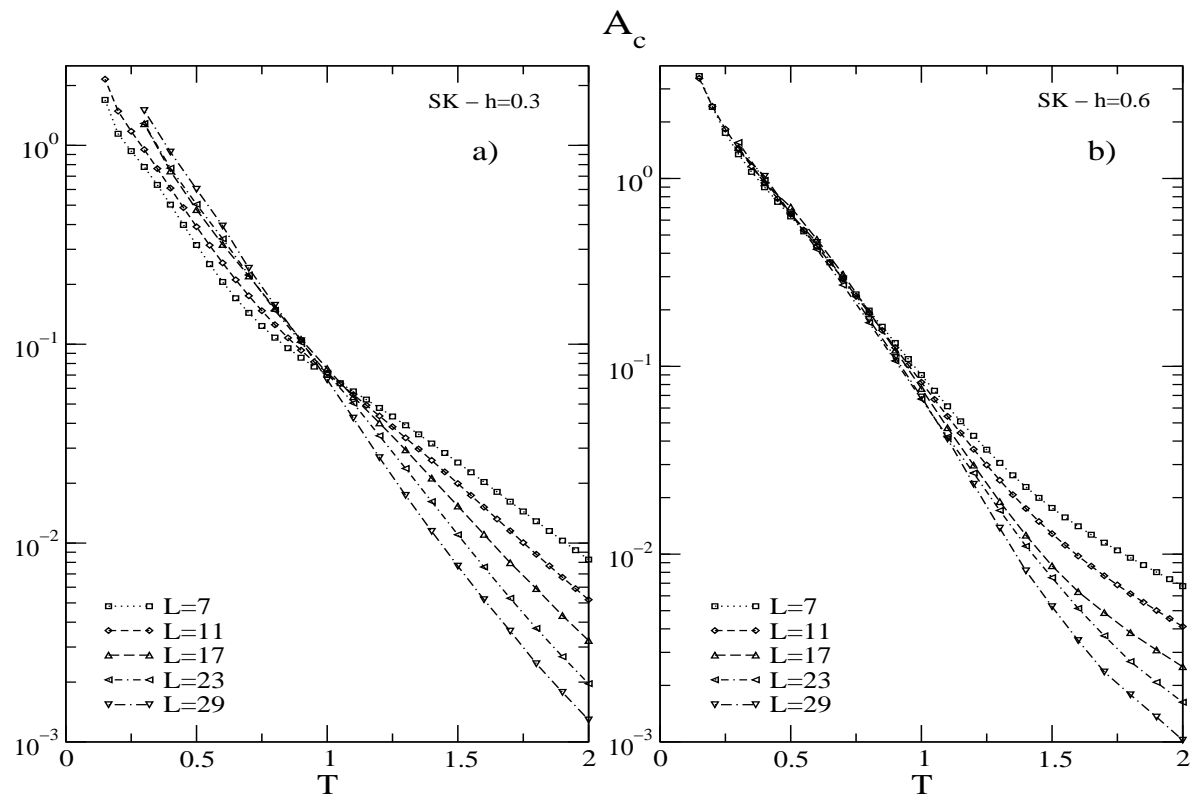

FIG. 7. $A_{c}$ for the SK model in applied field for sizes $N=7,11,13,23,29$ : (a) for $h=0.3$, (b) for $h=0.6$. 

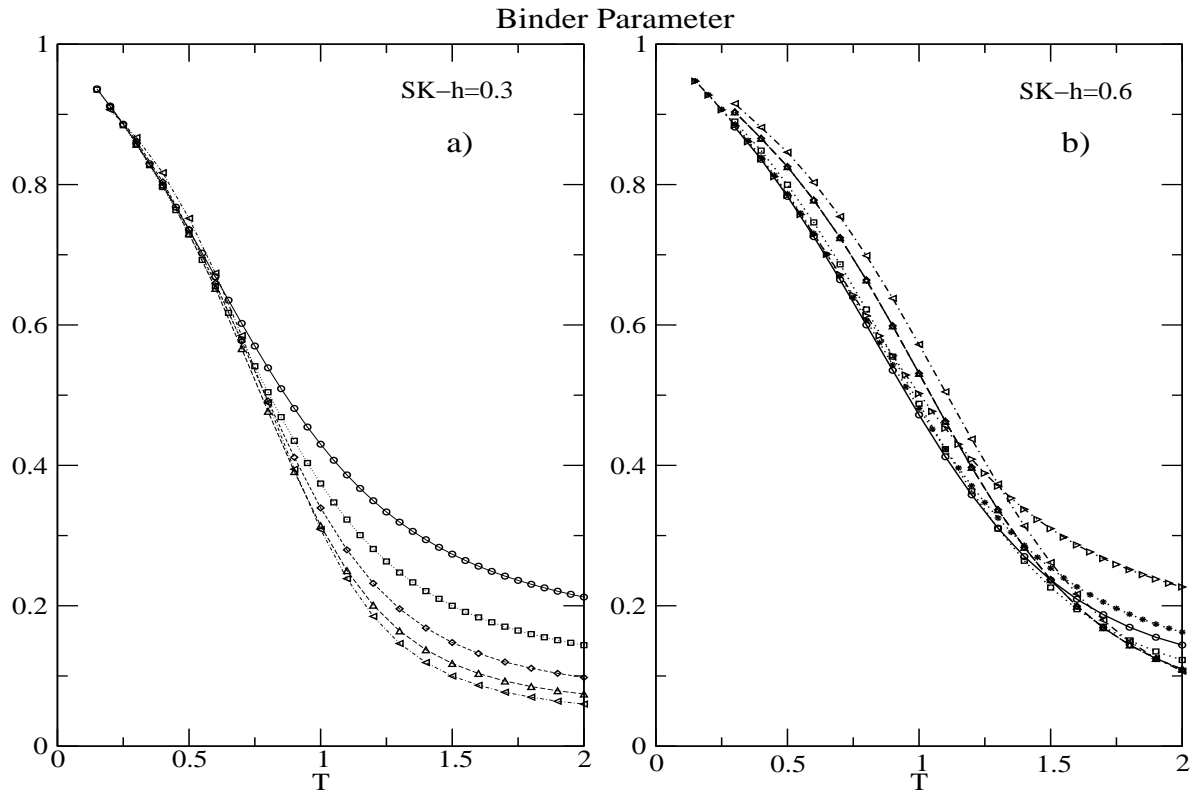

FIG. 8. Binder ratio, $B$, for the SK model in applied field for sizes $N=7,11,13,23,33$, (a) $h=0.3$ and (b) $h=0.6$.

$\mathrm{B}_{\mathrm{c}}$ (Binder connected ratio)
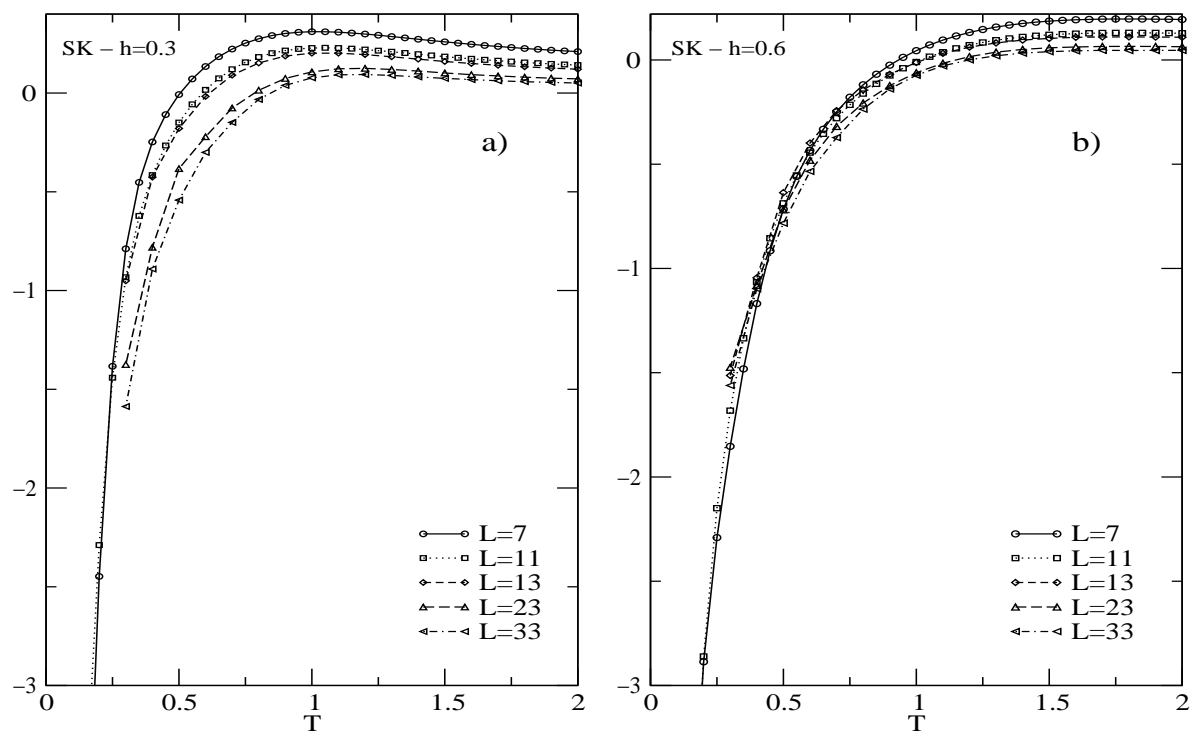

FIG. 9. Binder connected ratio, $B_{c}$, for the SK model in applied field for sizes $N=7,11,13,23,33$, with $h=0.3$ on the left and $h=0.6$ on the right.

\section{Summary of the results}

- SK at zero field: $A$ and $G$ are good parameters to locate the transition. Finite-size effects are evidenced by a crossing at a temperature slightly above $T_{c}$, that moves towards $T_{c}$ as size increases. The location of the transition point is more precisely located by $G$ and $A$ than by the Binder ratio, $B$. Finite-size scaling analysis reveals that $B, G$ and $A$ have the same scaling exponents, thus suggesting that the scaling behavior of $A$ and $G$ for generic short-range systems may be obtained from the standard renormalization group approach. 
- SK in a field: In general connected quantities, $G_{c}, A_{c}$ and $B_{c}$ show a crossing which gives evidence for a transition. Finite-size corrections to $T_{c}$ are of the same sign as those in the case $h=0$. Fipite-size effects are stronger at low fields because small systems are strongly affected by the $h=0$ fixed pointe29, suggesting that transitions in a field need to be studied at high magnetic fields.

- Continuous RSB: In the frozen phase and for the largest sizes, the general temperature behavior of the different quantities, $A, B$, and $G_{c}, A_{c}$ and $B_{c}$ in models in a field, is the same with and without a field. Therefore, it corroborates our results of the previous section: systems exhibiting the same kind of RSB below the transition (as in these two systems -the SK model with and without a field- which both display full-step RSB), display qualitatively the same behavior for $A, B, G_{c}, A_{c}$ and $B_{c}$. Note that we have intentionally excluded $G$ from the list since it always takes the value (for $V \rightarrow \infty$ ) $1 / 3$ in the low-temperature phase, hence does not depend on the particular RSB pattern.

\section{ISING P-SPIN, $\mathbf{P}=3$}

In this section we turn our attention to a model without time-reversal symmetry where OPF are finite. We have studied the mean-field $\mathrm{p}$-spin $(\mathrm{p}=3)$ model with Ising spins defined by:

$$
\mathcal{H}=-\sum_{i 1, i 2, i 3} J_{i_{1}, i_{2}, i_{3}} \sigma_{i_{1}} \sigma_{i_{2}} \sigma_{i_{3}}
$$

where couplings are Gaussian variables with zero mean and variance $\frac{3 !}{2 N^{3}}$. This model is well-known to have a one-step RSB transition at $T_{c} \approx 0.6$ showing a discontinuity in the order parameter, which at the transition point jumps from zero to a finite value. These kind of transitions are driven by a collapse of the configurational entropy (also called entropy crisis), and have been the object of a deep study as they have many points in common with the glass transition in structural glasse 32 . Particularly, we are interested in the fact that a measure of the violation of FDT in glasses can give us information on the pattern of RSB, i.e. on the parameter $m$ which determines the structure of the replica matrix $Q_{a b}$.

The interest of this model relies not only on the fact that it does not contain TRS in the Hamiltonian, but also on the fact that, as far as OPF do not vanish, results obtained in section III for the one-step RSB hold for this system and, thus can provide us with a direct check of the validity of identities (18), (19) and (20) for the finite $T$ behavior of $G_{c}, A_{c}$ and $B_{c}$ respectively. Moreover, this model presents a further simplification since $q_{0}$, the off-diagonal block value of the replica matrix element $Q_{a b}$, vanishes below the transition. For this reason, expressions (79) and (80) given in Appendix B for $A$ and $B$ have a much simpler expression only in terms of $m$ :

$$
\begin{aligned}
& A=\frac{m}{3(1-m)}, \\
& B=\frac{2-3 m}{2(m-1)} .
\end{aligned}
$$

In order to study the behavior of OPF in this model, we have made exact numerical computations of the partition function for sizes ranging from $N=4$ up to 11 averaging over 10000 samples and Monte Carlo simulations using the parallel tempering technique for $N=13,17,23,29,33$ averaging over $150-500$ samples. Results for $B, G_{c}, G$ and $A$ are displayed in figure 10 and for $A_{c}$ and $B_{c}$ in figure 11, the full line corresponding in each case to the theoretical prediction obtained by numerically solving the saddle point equations. We have to point out that despite the low number of samples due to the hard task of thermalizing these systems, results are strikingly good.

Our results for the Binder parameter (figure 10) do not display any crossing but show that around the transition temperature, $T_{c}=0.6, B$ becomes negative, and has a minimum which grows together with the size of the system as is expected from expression (24) which indicates that at the transition, where $m$ is set to one, the Binder ratio has a negative divergence. Results obtained by Hukushima and Kawamura in 3 for the Potts model, are different ( $B$ is expected to take the value -1 at the transition), since the actual values of $q_{1}, q_{0}$ and $m$ are different, thus corroborating our result for $B$ obtained in Appendix B (see eq. 80), which states that $B$ has a strong dependence on the model. Results for $B_{c}$ are very alike, since there is no crossing, and there is also a minimum around $T_{c}$ which deepens with size. This behavior is very well understood by looking at the infinite volume behavior of both parameters in figures 10 (b) and 11 (a) in which curves for different sizes approach to the theoretical curve from top to bottom and this is why there is no possible crossing. 
Instead, our results for $G_{c}, G, A$ and $A_{c}$ exhibit a crossing which moves from higher to lower temperatures giving evidence for the existence of the transition. As we had noted in the previous section, we observe that finite-size corrections to $T^{*}$ are bigger in disconnected averages than in connected ones, the formers giving a better estimate of $T_{c}$. However, it is important to note that the position of the maximum of $A$ which accounts for the existence of a phase where replica symmetry is broken has a quick saturation towards $T=0.6$, and grows with size as is expected from the divergence that this parameter shows at the transition (23). A similar situation is met in results for $A_{c}$ (figure 11), since there is also a predicted divergence at $m=1$, which is numerically observable by a maximum around $T_{c}$ which grows and sharpens with size.

From results for $G_{c}$, we can still go a bit further. We observe that, as the size increases, the shape of the curve approaches the one of the thermodynamic limit much faster than in the other parameters, due to the softer behavior of $G_{c}$. It is interesting to note that even though we see a crossing of the curves which moves toward $T_{c}$ from higher temperature as the number of spins increases, much more clear evidence for the existence of the transition and the kind of transition (i.e. one-step RSB) come from the existence of a bump which becomes closer to $T_{c}$ as the size grows. We observe that at low-temperatures curves always remain under the infinite-volume line, so that there is no crossing in the low $T$ phase. Moreover, curves quickly stuck to the value of the minimum displayed by the thermodynamic value of $G_{c}$. As we noted in the previous section, the fact that this value is universal can yield valuable information about the low-temperature phase of systems exhibiting one-step RSB.

Results in the former section showed that connected quantities, $G_{c}, A_{c}$ and $B_{c}$ for the SK model (figures 5 , 6, 7, 9) exhibited quite a different behavior, as the curves were smooth and did not show any concavity, nor divergence at the transition temperature. Hence, in addition to showing what kind of information we can extract from the behavior of $G_{c}, A_{c}, B_{c}, A$ and $B$, we also give clear quantitative evidence of how different RSB transitions look like extending the previous comparative analysis made in 3 between the 3-Potts model and the SK with no field.

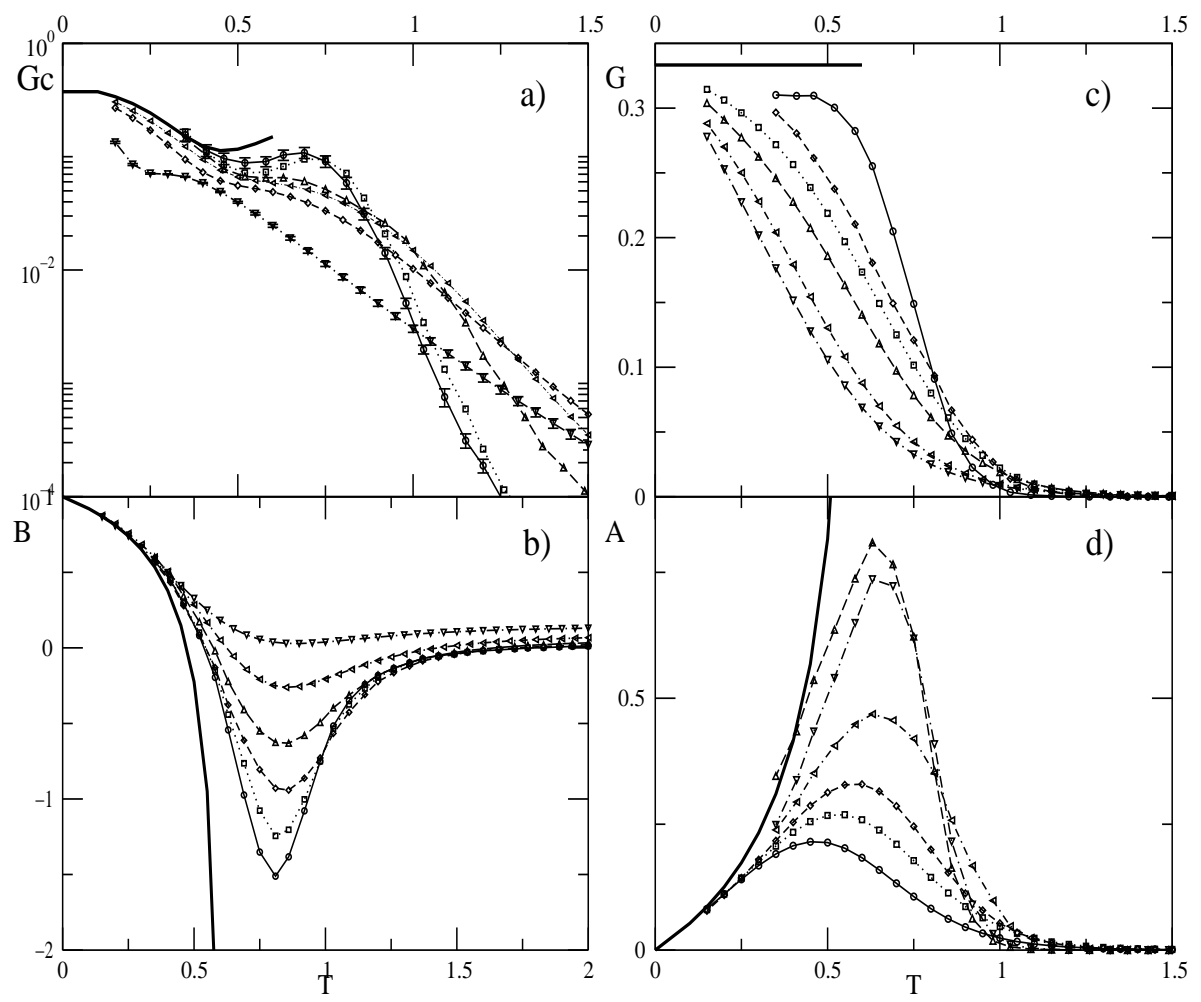

FIG. 10. We show the results for the 3-spin model for sizes ranging from $N=4$ to $N=33$. In every plot, the solid line corresponds to the theoretical prediction of expressions (14), (18), (79), and (80) for $G, G_{c}, A$ and $B_{c}$ respectively (see text). On the left side we display, in the top plot, results for $G_{c}$ for sizes $N=4,7,11,17,29,33$ from top to bottom in the low $T$ region, errors bars are showed for the larger and smaller sizes. Below we show the results for $B$ (Binder ratio) for sizes $N=7,11,17,23,29,33$ from bottom to top . On the right side, we show results for the non-connected averages, $A$ for sizes $N=5,8,11,17,29,33$ and $G$ for sizes $N=4,8,11,17,29$ both from bottom to top in the low-temperature region. 

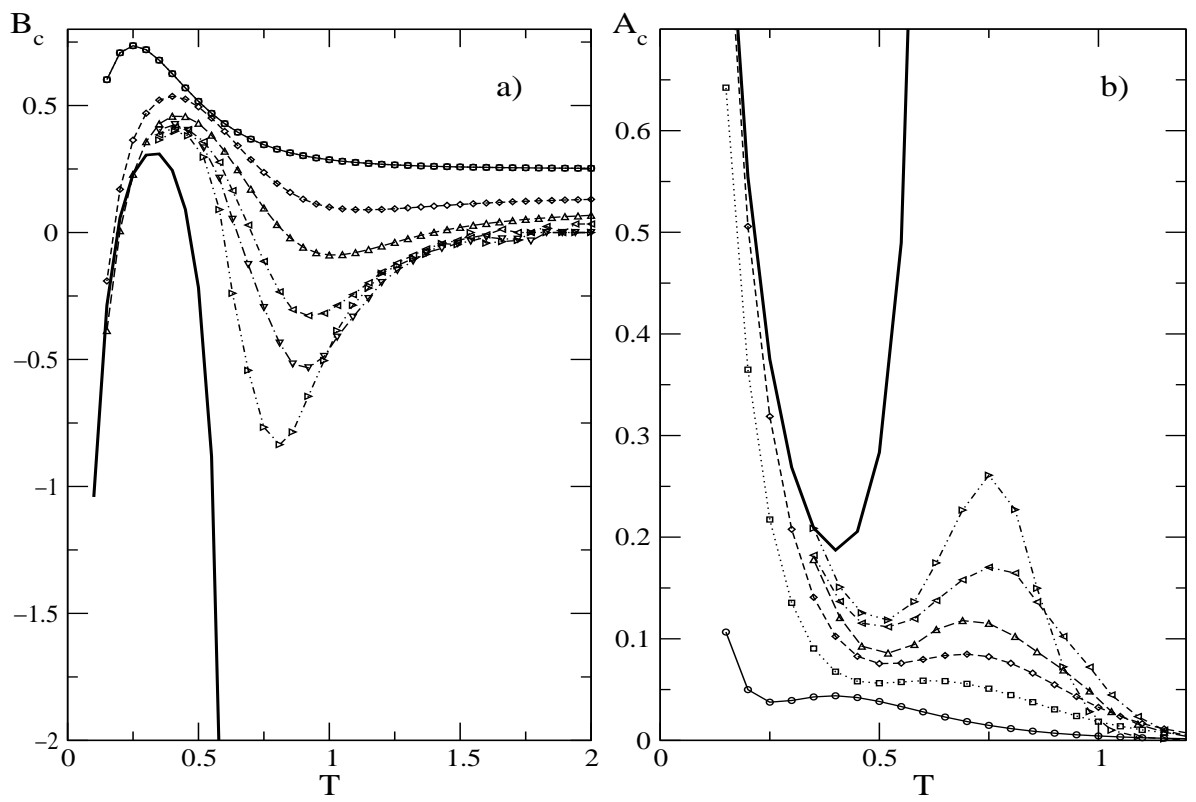

FIG. 11. Results for the 3-spin model: (a) $B_{c}$ for sizes $N=4,7,11,17,23,29$ from top to bottom, and (b) $A_{c}$ for sizes $N=4,7,11,17,23,29$ from bottom to top (in the low $T$ region). The solid line corresponds to the numerically solved theoretical prediction for $A_{c}$ and $B_{c}$, see eqs. (19) and (20) respectively.

\section{CONCLUSIONS}

In this paper we have presented a detailed analysis of parameters measuring OPF, $A, A_{c}, G$ and $G_{c}$, as well as $B$ and $B_{c}$, for small systems. Despite the numerical effort that computation of these parameters may imply, we have shown the powerfulness of these parameters and what kind of information we can extract from them. Our analysis through replica equivalence in section III, shows that the intimate connection of OPF with RSB yields a dependence of $A, B$, $G_{c}, A_{c}$ and $B_{c}$ on the RSB pattern, in opposition to $G$, which in the SG phase takes the value $1 / 3$ at $T<T_{c}$ ( even in marginal phases where OPF vanish, as stated in RS). Thus, $A, B, G_{c}, A_{c}$ distinguish between systems displaying different kinds of RSB in its frozen phase. Moreover, we have established a direct connection with glasses, since we have shown that at any temperature below $T_{c}$, connected quantities depend exclusively on $m$ (which determines the structure of the replica matrix $Q_{a b}$ ), which is the parameter having the physical meaning of an effective temperature which controls the violation of FDT in structural glasses.

We have analyzed mean-field models which exhibit time-reversal symmetry in their Hamiltonian and models which do not have this symmetry. The universality of $G$ and $G_{c}$ at zero temperature stated in RS has been checked and has been extended to the rest of quantities under study $A, A_{c}$ and $B_{c}$ and we have provided with a meaningful example of when identities (7) and (8) do not hold, i.e. the SK model in a field for small samples with an even number of spins. We have given many evidences that these parameters are useful tools to locate phase transitions into the spin-glass phase in any case. We can enumerate the main conclusions,

1. Advantage of $A, G(\mathrm{OPF})$ compared to $B$ (non $\mathrm{OPF}$ ). For models where an analysis of the Binder cumulant is enough to show the existence of a transition, i.e. the SK model without a field addressed in section IV, we have shown that already for very small samples, $G$ and $A$ give evidence for the existence of this transition. In other words, parameters measuring OPF have smaller finite-size corrections, so that we can extract useful information with no need of going to large systems. Moreover we have shown that $A$ and $G$ verify scaling relations and that the exponents coincide with those of the Binder ratio. Obviously, $A, G$ require more computational effort than $B$ but this effort compensates for the smallness of the corrections.

2. Advantage of connected parameters compared to disconnected ones. For models with TRS only $A, G, B$ are feasible to be computed. The interest of parameters involving connected averages concerns models which do not display TRS such as the SK model in a field and the Ising 3-spin model. For those cases we have once more checked the usefulness of these parameters to show the existence of a transition. A comparative analysis of $G$ and $G_{c}$ has shown that finite-size corrections are smaller for the latter, thus suggesting that connected 
parameters such as $G_{c}, A_{c}$ and $B_{c}$ are the best to look at in situations where there is no time-reversal symmetry. Our results for the SK model in a field show that the effects of the $h=0$ fixed point on small sizes are very strong (in agreement with the conventional wisdom 29.11), indicating the necessity to go to low temperaturesand high fields to show the existence of a spin-glass transition in a field. In view of some recent numerical result.33, 34 this goal is probably not out of reach.

3. Temperature dependence of OPF parameters on the RSB pattern. By studying these mean-field models which have different kinds of RSB transitions, we have checked that in systems where OPF do not vanish (i.e. when the results obtained through replica equivalence analysis are meaningful), $G_{c}, A_{c}, B_{c}, A$ and $G$ have a different behavior in the low-temperature phase. In addition, we have shown that in the 3 -spin model, which has a discontinuous transition, already for very small samples, $G_{c}$ yields information about the infinite-volume behavior obtained in section III (eq. 18) and thus about the replica symmetry breaking pattern through the temperature dependence of the parameter $m$.

To sum up, our results for $G, G_{c}, A$ and $A_{c}$ for small systems, in addition to what had already been stated in RS, i.e. the universal values at $T=0$ for both $G$ and $G_{c}$, and the trivial behavior of $G$ for any system having a SG transition, establish that parameters measuring OPF meet all the requirements that a proper parameter to locate phase transitions should have in disordered systems. We have studied in detail their behavior in systems where OPF do not vanish and have shown what kind of information small samples give about the infinite-volume behavior at finite temperature. In conclusion, the study of parameters measuring OPF opens a new direction in the study of the SG phase, as they are useful not only to locate the transition, but also to establish the nature of spin-glasses at finite temperature. Moreover, the scaling analysis reveals that there might be a connection between OPF and critical fluctuations that still lacks theoretical insight. The understanding of the trivial behavior of $G$ in the infinitevolume limit can give us more information about the mechanisms that control this frozen SG phase and can help us to understand the connection between spin-glasses having a marginally stable replica symmetric phase and systems having a broken SG phase. The further extension of these ideas to non-disordered glass models is also an interesting open problem.

Acknowledgments: F. R. is supported by the Ministerio de Educación y Ciencia in Spain (PB97-0971). M. S. is supported by the Ministerio de Educación y Ciencia of Spain, grant AP-98 36523875. M. P. and F. R. acknowledge financial support by the French-Spanish Picasso program (Acciones Integradas HF1998-0097).

Appendix A: Low-temperature behavior of $A, G, B$ and $A_{c}, G_{c}, B_{c}$.

In this Appendix we give a brief summary of the main points of the proof of the conjecture presented in RS, and show how the zero temperature values of all the parameters introduced in section II are obtained. We consider the most general finite spin system of Ising variables, described by the local field Hamiltonian:

$$
\mathcal{H}=-\sum_{i} h_{i} \sigma_{i}
$$

under two hypothesis:

- it has a unique ground state (up to a global flip of all the spins), given by the configuration $\left\{\sigma^{*}\right\}: E_{0}=$ $-\sum_{i} h_{i}^{*} \sigma_{i}^{*}$

- its local field probability distribution has a finite weight at zero field.

Under these two assumptions it was shown in RS that the low-temperature behavior of all the terms which appear in the definition of $G$ and $G_{c}$ (and hence in all the other quantities defined in section II) are dominated by one spin excitations 35 . This is because one-spin excitations yield a linear contribution in $T$, while two spin excitations yield a contribution of order $\left(T^{2}\right)$. Thus, in any general finite system where all excitations are possible, one-spin excitations give the dominant contribution at low $T$ and are responsible for the universal values $G=\frac{1}{3}$ and $G_{c}=\frac{13}{31}$ at $T=0$. In cases where the lowest excitation systematically involves clusters of spins a generalization of the present argument shows that the present results still hold 36 .

The argument proceeds as follows. First of all we note that all elements appearing in the definition of $G$ and $A$ can be expressed in terms of the two and four point correlation functions. If we define $T_{i j}=\left\langle\sigma_{i} \sigma_{j}\right\rangle^{2}$ and $T_{i j k l}=\left\langle\sigma_{i} \sigma_{j} \sigma_{k} \sigma_{l}\right\rangle^{2}$, then we have: 


$$
\begin{gathered}
\left\langle q^{2}\right\rangle=\frac{1}{V}+\frac{1}{V^{2}} \sum_{i \neq j} T_{i j}, \\
\left\langle q^{2}\right\rangle^{2}=\frac{1}{V^{2}}+\frac{2}{V^{3}} \sum_{i \neq j} T_{i j}+\frac{2}{V^{4}} \sum_{i \neq j} T_{i j}^{2}+\frac{4}{V^{4}} \sum_{(i \neq j \neq k)} T_{i j} T_{i k}+\frac{1}{V^{4}} \sum_{(i \neq j \neq k \neq l)} T_{i j} T_{k l}, \\
\left\langle q^{4}\right\rangle=\frac{1}{V^{4}}\left(3 V^{2}-2 V+(6 V-8) \sum_{i \neq j} T_{i j}+\sum_{(i, j, k, l)} T_{i j k l}\right) .
\end{gathered}
$$

Terms appearing in the definitions of the connected quantities also depend on one and three points correlation functions. With the definitions $T_{i}=\left\langle\sigma_{i}\right\rangle^{2}$ and $T_{i j k}=\left\langle\sigma_{i} \sigma_{j} \sigma_{k}\right\rangle^{2}$, we obtain the following expressions :

$$
\begin{gathered}
\langle q\rangle^{4}=\frac{1}{V^{4}}\left(\sum_{i} T_{i}^{4}+3 \sum_{i \neq j} T_{i}^{2} T_{j}^{2}+4 \sum_{i \neq j} T_{i}^{3} T_{j}+6 \sum_{(i, j, k)} T_{i}^{2} T_{j} T_{k}\right), \\
\left\langle q^{3}\right\rangle\langle q\rangle=\frac{1}{V^{4}}\left((3 V-2) \sum_{i} T_{i}^{2}+(3 V-2) \sum_{i \neq j} T_{i}^{2} T_{j}^{2}+3 \sum_{(i, j, k)} T_{i} T_{i j k}+\sum_{(i, j, k, l)} T_{i, j, k} T_{l}\right), \\
\left\langle q^{2}\right\rangle\langle q\rangle^{2}=\frac{1}{V^{4}}\left(V \sum_{i} T_{i}^{2}+V \sum_{i \neq j} T_{i} T_{j}+\sum_{(i, j, k)} T_{i}^{2} T_{j k}+4 \sum_{i \neq j} T_{i} T_{j} T_{i j}+2 \sum_{i \neq j} T_{i}^{2} T_{i j}+\sum_{(i, j, k, l)} T_{i, j} T_{k} T_{l}\right) .
\end{gathered}
$$

\section{One-spin excitations.}

We consider now all possible one-spin excitations. The energy cost of reversing the spin $i$ will be: $e_{i}=2 h_{i}^{*} \sigma_{i}^{*}$. From here one can compute the correlation functions previously introduced and obtain:

$$
\begin{gathered}
\left\langle\sigma_{i}\right\rangle=\sigma_{i}^{*}\left(1-\frac{2 \exp \left(-\beta e_{i}\right)}{1+\sum_{l=1, v} \exp \left(-2 \beta e_{l}\right)}\right) \approx \sigma_{i}^{*}\left(1-2 x_{i}\right), \\
\left\langle\sigma_{i} \sigma_{j}\right\rangle \approx \sigma_{i}^{*} \sigma_{j}^{*}\left(1-2\left(x_{i}+x_{j}\right)\right) \\
\left\langle\sigma_{i} \sigma_{j} \sigma_{k}\right\rangle \approx \sigma_{i}^{*} \sigma_{j}^{*} \sigma_{k}^{*}\left(1-2\left(x_{i}+x_{j}+x_{k}\right)\right) \\
\left\langle\sigma_{i} \sigma_{j} \sigma_{k} \sigma_{l}\right\rangle \approx \sigma_{i}^{*} \sigma_{j}^{*} \sigma_{k}^{*} \sigma_{l}^{*}\left(1-2\left(x_{i}+x_{j}+x_{k}+x_{l}\right)\right),
\end{gathered}
$$

with the definition $x_{i} \equiv \exp \left(-\beta e_{i}\right)$. Note that we have made the approximation $x_{i}<<1$, thus we can approximate the denominator in (32) by one. We have to stress that, as widely discussed in RS, this approximation implies that we are in the limit $\beta V<<1$. This is a key point in our argument concerning the local field probability distribution.

When performing the averages over the disorder one has to tackle with the following objects:

$$
\overline{x_{i}^{m} x_{j}^{n}} \equiv \int d h_{i} d h_{j} \hat{P}\left(h_{i}, h_{j}\right) e^{-2 \beta\left(m h_{i}^{*} \sigma_{i}^{*}+n h_{j}^{*} \sigma_{j}^{*}\right)}
$$

where the probability distribution of two fields results from integrating out the rest of the fields:

$$
\hat{P}\left(h_{i}^{*}, h_{j}^{*}\right) \equiv \int \prod_{k \neq i, j} d h_{k}^{*} P\left(h_{1}^{*}, \ldots, h_{V}^{*}\right)
$$

We have to point out that two local fields may be correlated so that the probability distribution of one field $\hat{P}\left(h_{i}^{*}\right) \equiv P_{i}$ depends on the site. However, the probability of having two equal local fields is very small 35 , thus in (37) there are no contributions of the type $\delta\left(h_{i}^{*}-h_{j}^{*}\right)$. It then follows from expression (36) by a simple saddle-point calculation 
that terms which depend on two sites have a contribution $\propto \mathcal{O}\left(T^{2}\right)$ while terms which depend only on one site have contributions $\propto \mathcal{O}(T):$

$$
\overline{x_{i}^{m} x_{j}^{n}}=\frac{T P_{i j}(0,0)}{m n} \quad \overline{x_{i}^{m}}=\frac{T P_{i}(0)}{m} .
$$

By looking at the above expressions for the correlation functions one observes that terms which depend on only one site are present only in one-spin excitations since, as shown in RS, correlation functions computed in the case of two-spin excitations depend on the products $x_{i} x_{j}$, thus bringing sub dominant contributions.

Then, after some algebra, the results obtained for the quantities appearing in the definitions of $G, G_{c}, A, A_{c}$ and $B$ are the following:

$$
\begin{aligned}
\overline{\left\langle q^{2}\right\rangle^{2}}-{\overline{\left\langle q^{2}\right\rangle}}^{2}=\frac{16 T \sum_{i} P_{i}(0)}{3 V^{4}}(V-1)^{2}+\mathcal{O}\left(T^{2}\right), \\
\overline{\left\langle q^{4}\right\rangle}-{\overline{\left\langle q^{2}\right\rangle}}^{2}=\frac{16 T \sum_{i} P_{i}(0)}{V^{4}}(V-1)^{2}+\mathcal{O}\left(T^{2}\right), \\
\overline{\left\langle(q-\langle q\rangle)^{2}\right\rangle^{2}}=\frac{218 T \sum_{i} P_{i}(0)}{105 V^{4}}+\mathcal{O}\left(T^{2}\right), \\
\overline{\left\langle(q-\langle q\rangle)^{2}\right\rangle}=\frac{8 T \sum_{i} P_{i}(0)}{3 V^{4}}+\mathcal{O}\left(T^{2}\right), \\
\overline{\left\langle(q-\langle q\rangle)^{4}\right\rangle}=\frac{1}{V^{4}} \frac{496 T \sum_{i} P_{i}(0)}{105 V^{4}}+\mathcal{O}\left(T^{2}\right) .
\end{aligned}
$$

Then we obtain for $G$ and $G_{c}$ for any finite volume,

$$
G=\frac{1}{3}+\mathcal{O}(T) \quad G_{c}=\frac{13}{31}+\mathcal{O}(T) .
$$

The situation is rather different for $A_{c}$ and $B_{c}$. From the expression of eq. (5) of $A_{c}$, we see that the numerator is linear in $T$, while the denominator ${\overline{\left\langle(q-\langle q\rangle)^{2}\right\rangle}}^{2}$ is quadratic in $T$. Hence we have that in the low-temperature limit:

$$
\lim _{T \rightarrow 0} A_{c} \propto \frac{1}{T} \rightarrow \infty
$$

The same situation occurs for the computation of $B_{c}$ given by eq. (何 where we have to compare $\overline{\left\langle(q-\langle q\rangle)^{4}\right\rangle} \propto T$ with ${\overline{\left\langle(q-\langle q\rangle)^{2}\right\rangle}}^{2} \propto T^{2}$. It then follows that:

$$
\lim _{T \rightarrow 0} B_{c} \propto-\frac{1}{T} \rightarrow-\infty .
$$

\section{Appendix B: Using replica equivalence to compute OPF.}

In this appendix we report explicit calculations of some results outlined in Section III. In particular, we describe in detail how to make use of the replica equivalence property which states that quantities such as $\sum_{a} Q_{a b}^{k}$ do not depend on the replica index $b$, to compute the different terms involved in $G$ and $G_{c}$ which lead to identities (8) and (18) respectively.

In general, we have to deal with terms involving one or more overlaps which in terms of the replica matrix elements $Q_{a b}$ read :

$$
\begin{gathered}
\overline{\left\langle q^{k}\right\rangle}=\frac{1}{n(n-1)} \sum_{a b} Q_{a b}^{k}=\int d q P(q) q^{k} \equiv q_{k} \\
\overline{\langle q\rangle^{y}}=\frac{\sum_{a, b, c, d \ldots, l_{2(y-1)}, l_{2 y-1}} Q_{a b} Q_{c d} . . Q_{l_{2 y-1}, l_{2 y}}}{n(n-1)(n-2) \ldots(n-(2 y-1))}=\int d q_{1} d q_{2} \ldots . . d q_{y} P\left(q_{1}, q_{2} \ldots . q_{y}\right) q_{1} q_{2} \ldots . . q_{y},
\end{gathered}
$$

where sums always run over different indexes. Note that we have defined $q_{k}$ in order to simplify the notation. As we have already said in section III this can also be expressed in terms of the $P(q)$ for a different number of replicas as 
we show in the last equality.

\section{Computation of $G$}

The terms which appear in the definition of $G$ are the following:

$$
\begin{aligned}
\overline{\left\langle q^{2}\right\rangle} & =\frac{1}{n(n-1)} \sum_{a b} Q_{a b}^{2}=\int d q P(q) q^{2} \equiv q_{2} \\
\overline{\left\langle q^{4}\right\rangle} & =\frac{1}{n(n-1)} \sum_{a b} Q_{a b}^{4}=\int d q P(q) q^{4} \equiv q_{4}, \\
\overline{\left\langle q^{2}\right\rangle^{2}} & =\frac{1}{n(n-1)(n-2)(n-3)} \sum_{a, b, c, d} Q_{a b}^{2} Q_{c d}^{2}=\int d q d q_{1} P\left(q, q_{1}\right) q^{2} q_{1}^{2}
\end{aligned}
$$

Thus we only have to deal with terms involving one and two overlaps. As is shown in 17 and discussed in detail in 18 , the RE property allows to express the probability distribution of two overlaps in terms of the probability distribution of one overlap: $P(q)$. Hence $\overline{\left\langle q^{2}\right\rangle^{2}}$ can be expressed in terms of $\overline{\left\langle q^{4}\right\rangle} \overline{\left\langle q^{2}\right\rangle}$ and $\overline{\langle q\rangle}$.

- Quantities involving two overlaps.

The general term reads :

$$
\overline{\left\langle q^{k}\right\rangle\left\langle q^{l}\right\rangle}=\frac{1}{n(n-1)(n-2)(n-3)} \sum_{a, b, c, d} Q_{a b}^{k} Q_{c d}^{l}
$$

The calculation proceeds as follows: the sum appearing in (52) can be re-expressed as:

$$
\sum_{a, b, c, d} Q_{a b}^{k} Q_{c d}^{l}=\sum_{a b} Q_{a b}^{k} \sum_{a b} Q_{a b}^{l}-2 \sum_{a b} Q_{a b}^{k+l}-4 \sum_{a, b, c} Q_{a b}^{k} Q_{a c}^{l}
$$

In order to compute the last term of the previous identity we use replica equivalence, i.e. :

$$
\sum_{c} Q_{a c}=\frac{1}{n} \sum_{a c} Q_{a c}
$$

Thus, for the last term in (53), we obtain:

$$
\sum_{a, b, c} Q_{a b}^{k} Q_{a c}^{l}=\sum_{a b} Q_{a b}^{k} \sum_{c} Q_{a c}^{l}=\sum_{a b} Q_{a b}^{k}\left(\frac{\sum_{a c} Q_{a c}^{l}}{n}-Q_{a b}^{l}\right),
$$

so that substituting this last result into identity (51) we obtain the following general relation:

$$
\begin{aligned}
\overline{\left\langle q^{k}\right\rangle\left\langle q^{l}\right\rangle} & =\frac{(n-4)(n-1)}{(n-2)(n-3)} \frac{\sum_{a b} Q_{a b}^{k}}{n(n-1)} \frac{\sum_{a b} Q_{a b}^{l}}{n(n-1)}+\frac{2}{(n-2)(n-3)} \frac{\sum_{a b} Q_{a b}^{k+l}}{n(n-1)} \\
& =\frac{(n-4)(n-1)}{(n-2)(n-3)} q_{k} q_{l}+\frac{2}{(n-2)(n-3)} q_{k+l} .
\end{aligned}
$$

¿From this relation, the computation of $\overline{\left\langle q^{2}\right\rangle^{2}}$ and terms such as $\overline{\langle q\rangle\left\langle q^{3}\right\rangle}$ and $\overline{\langle q\rangle^{2}}$ which appear in the definition of $G_{c}$ is straightforward. Thus for $\overline{\left\langle q^{2}\right\rangle^{2}}$ we obtain:

$$
\begin{aligned}
\overline{\left\langle q^{2}\right\rangle\left\langle q^{2}\right\rangle} & =\frac{(n-4)(n-1)}{(n-2)(n-3)}\left(\frac{\sum_{a b} Q_{a b}^{2}}{n(n-1)}\right)^{2}+\frac{2}{(n-2)(n-3)} \frac{\sum_{a b} Q_{a b}^{4}}{n(n-1)} \\
& =\frac{(n-4)(n-1)}{(n-2)(n-3)} q_{2}^{2}+\frac{2}{(n-2)(n-3)} q_{4} .
\end{aligned}
$$


At this point we are able to compute the value of the numerator and denominator of $G$, which yield the well-known result:

$$
\begin{aligned}
\text { numerator }= & \frac{2}{(n-2)(n-3)}\left(\int d q P(q) q^{4}-\left(\int d q P(q) q^{2}\right)^{2}\right) \\
\text { denominator }= & \int d q P(q) q^{4}-\left(\int d q P(q) q^{2}\right)^{2} \\
& \rightarrow \lim _{n \rightarrow 0} G=\frac{\text { numerator }}{\text { denominator }}=\frac{1}{3},
\end{aligned}
$$

provided $\operatorname{OPF}\left(\int d q P(q) q^{4}-\left(\int d q P(q) q^{2}\right)^{2}\right)$ do not vanish. For the parameter $A$ this yields in the limit $n \rightarrow 0$ :

$$
A=\frac{q_{4}-q_{2}^{2}}{3 q_{2}^{2}}
$$

\section{Computation of $G_{c}$}

Now we have to deal with harder terms which appear in the calculation of $G_{c}$ and involve the probability distribution of three and four overlaps. The numerator reads: $\overline{\left\langle q^{2}\right\rangle^{2}}-2 \overline{\left\langle q^{2}\right\rangle\langle q\rangle^{2}}+\overline{\langle q\rangle^{4}}-\left(\overline{\left\langle q^{2}\right\rangle}-\overline{\langle q\rangle^{2}}\right)^{2}$ and the denominator reads: $\overline{\left\langle q^{4}\right\rangle}+6 \overline{\left\langle q^{2}\right\rangle\langle q\rangle^{2}}-3 \overline{\langle q\rangle^{4}}-4 \overline{\left\langle q^{3}\right\rangle\langle q\rangle}-\left(\overline{\left\langle q^{2}\right\rangle}-\overline{\langle q\rangle^{2}}\right)^{2}$ so that we have to compute:

$$
\begin{array}{r}
\overline{\left\langle q^{2}\right\rangle\langle q\rangle^{2}}=\frac{1}{n(n-1)(n-2)(n-3)(n-4)(n-5)} \sum_{a, b, c, d, e, f} Q_{a b}^{2} Q_{c d} Q_{e f} \\
\overline{\langle q\rangle^{4}}=\frac{1}{n(n-1)(n-2)(n-3)(n-4)(n-5)(n-6)(n-7)} \sum_{a, b, c, d, e, f, g, h} Q_{a b} Q_{c d} Q_{e f} Q_{g h} .
\end{array}
$$

As we have already pointed in section III, these terms will depend in general in terms containing $P(q)$ and terms which depend on the probability distribution of 3 or 4 connected overlaps, terms like $\sum_{a, b, c} Q_{a b}^{k} Q_{b c}^{l} Q_{c a}^{p}$ and $\sum_{a, b, c, d} Q_{a b}^{k} Q_{b c}^{l} Q_{c a}^{p} Q_{c a}^{t}$ where replica equivalence cannot be used since we have in general that: $\sum_{b} Q_{a b}^{k} Q_{b c}^{l}$ depends on the replica indices $a$ and $c$, so that for any particular structure of the replica matrix $Q_{a b}$ will have different values.

- Quantities involving three overlaps.

Now we will derive the general relation for any term containing three overlaps such as (60). The general term is:

$$
\sum_{a, b, c, d, e, f} Q_{a b}^{k} Q_{c d}^{l} Q_{e f}^{m}=\sum_{a, b, c, d} Q_{a b}^{k} Q_{c d}^{l} \sum_{e, f} Q_{e f}^{m}-4 \sum_{a, b, c, d, e} Q_{a b}^{k} Q_{c d}^{l} Q_{e b}^{m}-4 \sum_{a, b, c, d, e} Q_{a b}^{k} Q_{c d}^{l} Q_{e c}^{m}
$$

The first term can be computed from identity (56), since we have:

$$
\frac{1}{n(n-1)(n-2)(n-3)(n-4)(n-5)} \sum_{(a, b, c, d} Q_{a b}^{k} Q_{c d}^{l} \sum_{e, f} Q_{e f}^{m}=\frac{n(n-1)}{(n-4)(n-5)} \overline{\left\langle q^{l}\right\rangle\left\langle q^{k}\right\rangle\left\langle q^{m}\right\rangle} .
$$

The other two terms on the r.h.s of relation (62) are identical by permuting sub-indices $(d, b)$ and super-indices $(l, k)$ so that we only have to compute one of them: $\sum_{a, b, c, d, e} Q_{a b}^{k} Q_{c d}^{l} Q_{e b}^{m}$. We can apply RE (54) to the sum of index $e$ and obtain:

$$
\sum_{a, b, c, d, e} Q_{a b}^{k} Q_{c d}^{l} Q_{e b}^{m}=\sum_{a, b, c, d} Q_{a b}^{k} Q_{c d}^{l}\left(\frac{\sum_{e, b} Q_{e b}^{m}}{n}\right)-\sum_{a, b, c, d} Q_{a b}^{k+m} Q_{c d}^{l}-2 \sum_{a, b, c, d} Q_{a b}^{k} Q_{c d}^{l} Q_{c b}^{m} .
$$

Again we make use of identity (56) to compute the two first terms on the r.h.s of the previous identity, so that we are only left with the last term: $\sum_{a, b, c, d} Q_{a b}^{k} Q_{c d}^{l} Q_{c b}^{m}$. By applying the RE to the sum over $d$ we finally get:

$$
\sum_{a, b, c, d} Q_{a b}^{k} Q_{c d}^{l} Q_{c b}^{m}=\sum_{a, b, c} Q_{a b}^{k} Q_{c b}^{m}\left(\frac{\sum_{d, c} Q_{d, c}^{l}}{n}\right)-\sum_{a, b, c} Q_{a b}^{k} Q_{c b}^{m} Q_{c a}^{l}-\sum_{a, b, c} Q_{a b}^{k} Q_{c b}^{m+l} .
$$


So, now, we know how to compute all the terms in function of the probability distribution of one overlap except for the term $\sum_{a, b, c} Q_{a b}^{k} Q_{c b}^{m} Q_{c a}^{l}$. Putting all terms together we obtain the following expression for the general element which involves the probability distribution of three overlaps,

$$
\begin{aligned}
\overline{\left\langle q^{k}\right\rangle\left\langle q^{l}\right\rangle\left\langle q^{m}\right\rangle} & =\frac{(n-4)(n-8)+8(n-1))(n-1)^{2}}{(n-2)(n-3)(n-4)(n-5)} \frac{\sum_{a b} Q_{a b}^{k}}{n(n-1)} \frac{\sum_{a b} Q_{a b}^{l}}{n(n-1)} \frac{\sum_{a b} Q_{a b}^{m}}{n(n-1)} \\
& +\frac{16 \sum_{a b} Q_{a b}^{l+m+k}-8(n-2) \sum_{a, b, c} Q_{a b}^{k} Q_{c b}^{m} Q_{c a}^{l}}{n(n-1)(n-2)(n-3)(n-4)(n-5)} \\
& +\frac{2(n-8)(n-1)}{(n-2)(n-3)(n-4)(n-5)}\left(\frac{\sum_{a b} Q_{a b}^{k+l}}{n(n-1)} \frac{\sum_{a b} Q_{a b}^{m}}{n(n-1)}+\frac{\sum_{a b} Q_{a b}^{m+l}}{n(n-1)} \frac{\sum_{a b} Q_{a b}^{k}}{n(n-1)}\right. \\
& +\frac{\left.\sum_{a b} Q_{a b}^{k+m} \sum_{a b} Q_{a b}^{l}\right)}{n(n-1)} \frac{(n-1)}{(n-4)(n-8)+8(n-1))(n-1)^{2}} \\
& =\frac{(n-2)(n-3)(n-4)(n-5)}{(n-8)(n-1)} q_{k} q_{l} q_{m} \\
& +\frac{16}{(n-2)(n-3)(n-4)(n-5)}\left(q_{k} q_{m+l}+q_{l} q_{m+k}+q_{m} q_{k+l}\right) \\
& +\frac{8(n-2)}{(n-2)(n-3)(n-4)(n-5)} q_{m+l+k}-\frac{8(n-2)(n-3)(n-4)(n-5)}{(n-1)} Q_{k, l, m}^{3} .
\end{aligned}
$$

Where we have introduced the following definitions:

$$
Q_{k, l, m}^{3} \equiv \int d q_{1} d q_{2} d q_{3} P\left(q_{1}, q_{2}, q_{3}\right) q_{1}^{l} q_{2}^{k} q_{3}^{m} \text { and } Q_{k, l, m, p}^{4} \equiv \int d q_{1} d q_{2} d q_{3} P\left(q_{a}, q_{b}, q_{c}, q_{d}\right) q_{a}^{l} q_{b}^{k} q_{c}^{m} q_{d}^{p} .
$$

Finally we get the following expression for the term $(60)$ :

$$
\begin{aligned}
\overline{\left\langle q^{2}\right\rangle\langle q\rangle^{2}} & =\frac{(n-4)(n-8)+8(n-1))(n-1)^{2}}{(n-2)(n-3)(n-4)(n-5)} q_{1}^{2} q_{2} \\
& +\frac{2(n-8)(n-1)}{(n-2)(n-3)(n-4)(n-5)}\left(q_{2}^{2}+2 q_{1} q_{3}\right) \\
& +\frac{16}{(n-2)(n-3)(n-4)(n-5)} q_{4}-\frac{8(n-2)}{(n-2)(n-3)(n-4)(n-5)} Q_{1,1,1}^{3} .
\end{aligned}
$$

For the other term,$\overline{\langle q\rangle^{4}}$, calculations require more effort since one has two deal with terms involving four overlaps, but the procedure is similar. We only quote the final result:

$$
\begin{aligned}
\overline{\langle q\rangle^{4}} & =\frac{\left((n-1)^{3}(-672+n(208+(n-24) n))\right.}{(n-2)(n-3)(n-4)(n-5)(n-6)(n-7)} q_{1}^{4}+\frac{12(n-1)^{2}(112+(n-20) n)}{(n-2)(n-3)(n-4)(n-5)(n-6)(n-7)} q_{1}^{2} q_{2} \\
& -\frac{32(n-12)(n-1)}{(n-2)(n-3)(n-4)(n-5)(n-6)(n-7)} q_{1}\left(-2 q_{3}+(n-2) Q_{1,1,1}^{3}\right) \\
& +\frac{12((n-16)(n-1)}{(n-2)(n-3)(n-4)(n-5)(n-6)(n-7)} q_{2}^{2}+\frac{20}{(n-2)(n-3)(n-4)(n-5)(n-6)(n-7)} q_{4} \\
& -\frac{4(n-2)}{(n-2)(n-3)(n-4)(n-5)(n-6)(n-7)}\left(8 Q_{2,1,1}^{3}-(n-3) Q_{1,1,1,1}^{4}\right) .
\end{aligned}
$$

Further calculations lead to a rather intricated expression for $G_{c}$ in the limit $n \rightarrow 0$ :

$$
G_{c}=\frac{49 q_{1}^{4}-77 q_{1}^{2} q_{2}+q_{2}^{2}+60 q_{1} q_{3}-18 q_{4}+18 Q_{2,1,1}^{3}-24 q_{1} Q_{1,1,1}^{3}-9 Q_{1,1,1,1}^{4}}{133 q_{1}^{4}-329 q_{1}^{2} q_{2}-38 q_{2}^{2}+240 q_{1} q_{3}-51 q_{4}-54 Q_{2,1,1}^{3}+72 q_{1} Q_{1,1,1}^{3}+27 Q_{1,1,1,1}^{4}} .
$$

¿From which the following results for $A_{c}$ and $B_{c}$ are obtained:

$$
A_{c}=\frac{-49 q_{1}^{4}+77 q_{1}^{2} q_{2}-q_{2}^{2}-60 q_{1} q_{3}+18 q_{4}-18 Q_{2,1,1}^{3}+24 q_{1} Q_{1,1,1}^{3}+9 Q_{1,1,1,1}^{4}}{70\left(q_{2}-q^{2}\right)^{2}},
$$




$$
B_{c}=3 \frac{91 q_{1}^{4}-203 q_{1}^{2} q_{2}+34 q_{2}^{2}+80 q_{1} q_{3}-17 q_{4}-18 Q_{2,1,1}^{3}+24 q_{1} Q_{1,1,1}^{3}+9 Q_{1,1,1,1}^{4}}{140\left(q_{2}-q^{2}\right)^{2}} .
$$

Thus, one has to go to particular structures of the replica matrix $Q_{a b}$ to try to obtain a simpler expression for $B, A$, $G_{c}, B_{c}$, and $A_{c}$.

\section{The symmetric case}

In the symmetric case, $B$, the Binder cumulant takes the value 1 in the frozen phase and $A$ vanishes since there are no OPF. In all the connected quantities, as well as in $G$, both numerator and denominator vanish, thus RE gives no information. Despite, we have to stress that, as shown numerically in RS for the spherical SK model, $G$ still takes the value $1 / 3$ in the $\mathrm{SG}$ phase.

\section{One-step replica symmetry breaking}

In this particular case the structure of the replica matrix is the following: replicas are distributed into $m$ blocks, so that the matrix elements can have two possible values $q_{1}$ if the two replicas belong to the same block and $q_{0}$ if they belong to different blocks. Thus, we have for the different terms which appear in the calculation of $G_{c}, A, A_{c}, B$ and $B_{c}$ :

$$
\begin{gathered}
\frac{1}{n(n-1)} \sum_{a, b} Q_{a b}^{k}=\frac{(m-1) q_{1}^{k}+(n-m) q_{0}^{k}}{n-1}, \\
\frac{1}{n(n-1)(n-2)} \sum_{a, b, c} Q_{a b} Q_{b c} Q_{c a}= \\
\frac{q_{1}(m-1)\left[q_{1}^{2}(m-2)+q_{0}^{2}(n-m)\right]+q_{0}(n-m)\left[2 q_{1} q_{0}(m-1)+q_{0}^{2}(n-2 m)\right]}{n-1}, \\
\frac{1}{n(n-1)(n-2)} \sum_{a, b, c} Q_{a b}^{2} Q_{b c} Q_{c a}= \\
\frac{q_{1}^{2}(m-1)\left[q_{1}^{2}(m-2)+q_{0}^{2}(n-m)\right]+q_{0}^{2}(n-m)\left[2 q_{1} q_{0}(m-1)+q_{0}^{2}(n-2 m)\right]}{n-1}, \\
\frac{1}{n} \sum_{a, b, c, d} Q_{a b} Q_{b c} Q_{c d} Q_{d a}=(m-1)\left((m-3)(m-2) q_{1}^{4}-2(3 m-5)(m-n) q_{1}^{2} q_{0}^{2}+4\left(2 m^{2}-3 m n+n^{2}\right) q_{1} q_{0}^{3}\right) \\
-\left(3 m^{2}+m(2-3 n)+(n-1)^{2}\right)(m-n) q_{0}^{4} .
\end{gathered}
$$

Substituting all these previous expressions into relations (69), (66), (68) yields, in the limit $n \rightarrow 0$ the expression already given in section III for the connected quantities $G_{c}$ (18), $A_{c}(19)$ and $B_{c}(20)$ :

$$
G_{c}=\frac{39-113 m+98 m^{2}}{93-221 m+266 m^{2}} \text {. }
$$

In the same way we obtain expressions for $A_{c}$ and $B_{c}$ which do not depend on the actual values of the elements of the replica matrix:

$$
A_{c}=\frac{39-113 m+98 m^{2}}{140 m(1-m)} \text { and } B_{c}=\frac{3\left(31-167 m+182 m^{2}\right)}{280 m(1-m)} .
$$

For the other disconnected quantities, $A$ and $B$ we obtain an expression which depends not only on $m$ but also on the elements of $Q_{a b}$, this is, $q_{0}$ and $q_{1}$. The expressions are the following:

$$
A=-\frac{(m-1) m\left(q_{1}^{2}-q_{0}^{2}\right)^{2}}{3\left((m-1) q_{1}^{2}-m q_{0}^{2}\right)^{2}}
$$




$$
B=\frac{(m-1) q_{1}^{4}-m q_{0}^{4}+3\left((m-1) q_{1}^{2}-m q_{0}^{2}\right)^{2}}{2\left((m-1) q_{1}^{2}-m q_{0}^{2}\right)^{2}}
$$

Comparing $A, B$ to the other connected parameters we find a further dependence on the parameters $q_{0}, q_{1}$. Computations for further steps of RSB are performed in the same way. However for a two-step patter for $Q_{a b}$, we do not obtain a simple expression from which it is possible to extract general conclusions.

${ }^{1}$ E. Marinari, C. Naitza, G. Parisi, M. Picco, F. Ritort and F. Zuliani, Phys. Rev. Lett. 81 1698 (1998); H. Bokil, A. J. Bray, B. Drossel and M. A. Moore, preprint cond-mat/9811080; E. Marinari, C. Naitza, G. Parisi, M. Picco, F. Ritort and F. Zuliani, Phys. Rev. Lett. 825175 (1999).

${ }^{2}$ F. Ritort and M. Sales, A conjectured scenario for order-parameter fluctuations in spin glasses, cond-mat/0003336 J. Phys. A (Math. Gen.) 331 (2000).

${ }^{3} \mathrm{~K}$. Hukushima and H. Kawamura, How the replica-symmetry-breaking transition looks like in finite-size simulations, preprint cond-mat/0003226.

4 M. Campellone, B. Coluzzi and G. Parisi, Phys. Rev. B 58, 12081 (1998).

${ }^{5}$ G. Parisi, M. Picco and F. Ritort, Phys. Rev. E 60, 58 (1999).

${ }^{6}$ H. G. Ballesteros, A. Cruz, L. A. Fernandez, V. Martin-Mayor, J. Pech, J. J. Ruiz-Lorenzo, A. Tarancon, P. Tellez, C. L. Ullod, C. Ungil, gond-mat/0006211.

${ }^{7}$ H. Bokil et al. int.

${ }^{8}$ H. Hukushima and K. Kawamura, Chiral-glass transition and replica symmetry breaking of three-dimensional Heisenberg spin glasses, preprint cond-mat/ 0001127 to appear in Pys. Rev. E.

${ }^{9}$ A. Pagnani, G. Parisi and F. Ricci-Tersenghi, Phys. Rev. Lett. 842026 (2000).

${ }^{10}$ M. Picco and F. Ritort, Physica A 25046 (1998).

${ }^{11}$ B. Drossel, H. Bokil, M. A. Moore, Spin glasses without time-reversal symmetry and the absence of a genuine structural glass transition, preprint cond-mat/0007315.

${ }^{12}$ H. G. Ballesteros, L. A. Fernandez, V. Martin-Mayor, A. Muñoz-Sudupe, G. Parisi and J. J. Ruiz-Lorenzo, Nucl.Phys. B512[FS], 681 (1998); Phys. Rev. B 58, 2740 (1998).

13 S. Wiseman and E. Domany, Phys. Rev. E 58, 2938 (1998).

14 A. Aharony and A. B. Harris, Phys. Rev. Lett. 773700 (1996).

${ }^{15}$ G. Parisi, J. Phys. A (Math. Gen.) 131101 (1980).

${ }^{16}$ A. J. Bray, M. A. Moore and A. P. Young, J. Phys. A 16, 2063 (1983).

17 M. Mézard, G. Parisi, N. Sourlas and M. A. Virasoro, J. Physique 45, 843 (1984).

${ }^{18} \mathrm{G}$. Parisi, On the probabilistic formulation of the replica approach to spin glasses, preprint cond-mat/9801081.

${ }^{19}$ M. Aizenmann, P. Contucci, J. Stat. Phys 92765 (1998).

${ }^{20}$ F. Guerra, Int. J. Mod. Phys. B 10, 1675 (1996).

${ }^{21}$ B. Coluzzi, E. Marinari, G. Parisi, H. Rieger, preprint cond-mat/0003287.

${ }^{22}$ E. Marinari, F. Zuliani, J. Phys A (Math. Gen.) 327447 (1999).

${ }^{23}$ A. P. Young and T. R. Kirkpatrick, Phys. Rev. B 25440 (1982).

${ }^{24}$ K. Hukushima and K. Nemoto, J. Phys. Soc. Jpn. 651604 (1996); K. Hukushima, H. Takayama, and H. Yoshino, J. Phys. Soc. Jpn. 6712 (1998).

${ }^{25}$ E. Marinari, G. Parisi, F. Ricci-Teresengh, J. Ruiz-Lorenzo, F. Zuliani, J. Stat. Phys. 98973 (2000).

${ }^{26}$ R. N. Bhatt and A. P. Young, Phys. Rev. B 375606 (1998).

${ }^{27}$ F. Slanina, G. Parisi and F. Ritort, J. Phys. A (Math. Gen.) 26247 (1993).

${ }^{28}$ M. Mézard, G. Parisi and M.A. Virasoro, Spin Glass Theory and Beyond. World Scientific (Singapore, 1987); K. Binder and A. P. Young, Rev. Mod. Phys. 58 (1986) 801; K. H. Fischer and J. A. Hertz, "Spin Glasses" Cambridge University Press, (1991).

${ }^{29}$ D. A. Huse, D. S. Fisher, J. Phys. I 1621 (1992).

${ }^{30}$ M. Picco, F. Ritort, J. Physique I 41618 (1994).

${ }^{31}$ B. Derrida, Phys. Rev. B 24 2613(1981); E. Gardner, Nucl. Phys. B 257747 (1985).

${ }^{32}$ T. R. Kirkpatrick, and D. Thirumalai, Phys Rev. Lett.58, 2091 (1987); T. R. Kirkpatrick, and P. G. Wolyness, in Phys. Rev. B 368552 (1987); For a recent review see M. Mézard, preprint cond-mat/0005173 and references therein.

${ }^{33}$ N. Hatano and J. E. Gubernatis, Evidence for the droplet picture in the $3 D \pm J$ spin glass, Preprint cond-mat/0008115

${ }^{34}$ H. G. Katzgraber, M. Palassini, A. P. Young, Spin Glasses at Low Temperatures, Preprint cond-mat/0007113 
${ }^{35}$ Calculations have been performed under the assumption that there are no singular correlations in the ground state local field distribution, which is a direct consequence of dealing with a finite volume system.

${ }^{36}$ F. Ritort and M. Sales (unpublished) 